\title{
Exotic bonding regimes uncovered in excited states
}

\author{
José Luis Casals Sainz, ${ }^{a}$ Jesús Jara-Cortés, ${ }^{b}$ \\ Jesús Hernández-Trujillo, ${ }^{c}$ José Manuel Guevara-Vela, ${ }^{a}$ \\ Evelio Francisco, ${ }^{a}$ Ángel Martín Pendás ${ }^{a *}$ \\ July 15,2019
}

\begin{abstract}
Real space tools are employed to show that the chemical bonding scenario used routinely to understand ground states lacks the necessary flexibility in excited states. We show that even for two-center, two-electron links the real space bond orders admit exotic values that have never been reported. The nature of these situations is uncovered by using electron counting techniques that provide an appealing statistical interpretation of bonding descriptors, together with simple physical models. Greater than one as well as negative bond orders for a single bonding electron pair are shown to emerge in situations where the electrons in the pair show a gregarious (bosonic) instead of the usual lonely (fermionic) behavior. In the first case the gregarious pair is intra-atomic, while the coupling is interatomic in the second. A number of examples are used to substantiate our claims.
\end{abstract}

Keywords: Chemical bonding, IQA, excited states, bonding regimes.

\footnotetext{
${ }^{[a]}$ Prof. Angel. Martín Pendás, Jose Luis Casals Sainz, Prof. Evelio Francisco.

Departamento de Química Física y Analítica.

Facultad de Química. Universidad de Oviedo.

33006-Oviedo. Spain.

E-mail: ampendas@uniovi.es

[b]Prof. Jesús Jara-Cortés.

Unidad Académica de Ciencias Básicas e Ingenierias, Universidad Autónoma de Nayarit, Tepic, 63155, México.

[c]Prof. Jesús Hernández-Trujillo.

Departamento de Física y Química Teórica, Facultad de Química, UNAM, México City, 04510, México.
} 


\section{Introduction}

In the last years, the study and characterization of excited states (ESs) has become an increasing area of interest due to its impact in a wide variety of fields including physics, chemistry, and biology. [1] The technological role of ESs is also growing. The near future high efficiency photovoltaic devices, for instance, depends on the ability to manipulate not well understood processes, like singlet fission. [2,3] Similarly, many biological processes involve light absortion and light harvesting. [4,5] In most cases, complicated setups, short life times, etc. make the experimental work on ESs difficult. $[4,6]$

Under such circumstances, the availability of accurate theoretical models and tools to study these states of matter becomes crucial. Recent methodological advances have gone a long way towards this end, and together with rigorous techniques, like the equation of motion (EOM) method and its variants, [7] lower cost procedures like the time-dependent density functional theory (TDDFT), [8] or the maximum overlap method (MOM), [9] have appeared. As it is usual in Chemistry, however, as more and more accurate computations become feasible, the more diffuse the relation between the results of calculations and the intuitive concepts of chemistry becomes, leaving room for completely unexpected results. This is particularly true when chemical meaning is extracted from calculations by using techniques explicitly taylored for ground states. An immediate example is the naïve molecular orbital definition of bond order as a difference of occupied bonding and antibonding states. As we move to ESs, where electron correlation is usually important, both the concept of occupied as well as of bonding or antibonding state vanish in thin air.

Real space techniques in the theory of chemical bonding [10] provide a solution to these problems by using orbital invariant bonding descriptors. As we show, exploring excited states with them considerably expands the chemical bonding scenario that is usually found in ground states.

In a previous work [11] we have already shown how to use the real space chemical bonding toolbox to provide a new interpretation of archetypal elementary photophysical processes from an energetic partitioning point of view. This was done by applying the interacting quantum atoms (IQA) formalism. $[12,13]$ Now we analyze the electronic redistribution accompanying excitation phenomena to uncover rather exotic bonding regimes not reported so far. We focus on how electrons localize and delocalize in space. Returning to the bond order concept, a number of works have 
shown that bond order becomes a measure of two-center electron delocalization when transported to real space, [14] and that it also conveys bond energy information. [15] A generalization of the real space bond order descriptors, known as delocalization indices (DIs), to many center cases is easy, [16-18] and multicenter delocalization indices (MCls) or bond orders have shown their power at rationalizing complex concepts such as aromaticity. [18-20]

The accessible numerical range of these descriptors is also known. For instance, the standard covalent bond order (DI) [21] in a two-center only, two-electron $(2 \mathrm{c}, 2 \mathrm{e})$ link must be positive and fulfill $0 \leq \mathrm{DI} \leq 2$. Standard chemical intuition assigns a maximum bond order of one to such situations and, in fact, no bonds with $\mathrm{DI}>1$ have been found in ground states. This is no longer true in ESs, and interesting new bonding situations may appear which are easily classified and, more importantly, rationalized, in real space. Similarly, when more than two centers exist, the DI may become negative. All these possibilities will be instantiated and examined here, for the first time.

To do so we have decided to rest on physical models that provide a clear picture of the meaning of these potentially mind-disturbing chemical situations. We have found that the Hubbard model, [22] both in its repulsive and attractive flavors, is particularly suited to that end, mimicking the qualitative behavior of actual computations. Although many space partitionings have been proposed in the literature, [13] we will stick to that provided by the Quantum Theory of atoms in molecules (QTAIM). [10]

We will first review briefly some basic aspects of our approach, including computational details of our calculations. Then we will discuss results in some simple excited states of $\mathrm{H}_{2}$, turning to excimer states in dihelium and charge transfer processes in $\mathrm{LiF}$ and $\mathrm{LiH}$. This provides a scaffold that is used to expand the landscape of bonding regimes by studying the $\mathrm{H}_{4}$ molecule in its $\mathrm{D}_{4 \mathrm{~h}}$ symmetry and the conical intersection in ethylene.

\section{Theoretical and methodological aspects}

Much as naïve bond orders are related to counting electrons in specific states, bond order in real space is interestingly linked to the statistics of electron counting, i.e. to the statistics of electron distribution functions (EDFs). We thus include a succinct review of these ideas, together with a minimal description of the Hubbard model. 


\section{Bond orders and electron counting}

An appealing picture of chemical bonding in terms of how the population of electrons in atoms fluctuates is being developed. [23,24] Given an N-electron molecule and an exhaustive (up to a null measure set) partition of the real space $\mathcal{R}^{3}$ into $\mathrm{m}$ regions $\Omega_{1}, \Omega_{2}, \ldots, \Omega_{\mathrm{m}}\left(\Omega_{1} \cup \Omega_{2} \cup \cdots \cup \Omega_{\mathrm{m}}=\mathcal{R}^{3}\right)$, the EDF is the statistical distribution function of the electron populations in the spatial regions, as described by the set of probabilities $p\left(n_{1}, n_{2}, \ldots, n_{m}\right)$ that exactly $n_{1}$ electrons lie in $\Omega_{1}, n_{2}$ electrons in $\Omega_{2}, \ldots$, and $n_{m}$ electrons in $\Omega_{m}$, the set $\left\{n_{p}\right\}$ being comprised of integers satisfying $n_{1}+n_{2}+$ $\cdots+n_{m}=N$. In more formal terms, a real space region may be understood as an open quantum system in a mixed state characterized by a fluctuating number of electrons, since the wavefunction of the total system is not an eigenstate of the operator defining the number of electrons in domain $\Omega_{\mathrm{i}}, \hat{\mathrm{N}}_{\mathrm{i}}$. This means that $\mathrm{N}_{\mathrm{i}}=\left\langle\hat{\mathrm{N}}_{\mathrm{i}}\right\rangle=\int_{\Omega_{\mathrm{i}}} \mathrm{d} \mathbf{r} \rho(\mathbf{r})$, the average number of electrons in $\Omega_{\mathrm{i}}$, is not an eigenvalue of $\hat{N}_{i}$, so that measuring the number of electrons in the domain will render values $n_{i}$ ranging from 0 to $\mathrm{N}$, the total number of electrons, with a defined set of probabilities, $p\left(n_{i}\right)$. This is the one-fragment EDF for domain $\Omega_{\mathrm{i}}$. In the general case, the multivariate electron distribution function $\mathrm{p}\left(\mathrm{n}_{1}, \mathrm{n}_{2}, \cdots, \mathrm{n}_{\mathrm{m}}\right)$ is used.

The $\Omega$ 3D domains can be arbitrary, but when using QTAIM atomic basins a partition of the $\mathrm{N}$ electrons of the molecule that assigns a given number of electrons to each of these regions, $\mathrm{S}\left(\mathrm{n}_{1}, \mathrm{n}_{2}, \ldots, \mathrm{n}_{\mathrm{m}}\right) \equiv \mathrm{S}\left(\left\{\mathrm{n}_{\mathrm{p}}\right\}\right)$, or simply $\left(\mathrm{n}_{1}, \mathrm{n}_{2}, \ldots, \mathrm{n}_{\mathrm{m}}\right) \equiv\left\{\mathrm{n}_{\mathrm{p}}\right\}$, is called a real space resonance structure (RSRS). If electrons are spin-seggregated, then we come to spin-resolved EDFs, and we use a set of probabilities $\mathrm{p}\left(\mathrm{n}_{1}^{\alpha}, \mathrm{n}_{1}^{\beta}, \mathrm{n}_{2}^{\alpha}, \mathrm{n}_{2}^{\beta}, \ldots, \mathrm{n}_{\mathrm{m}}^{\alpha}, \mathrm{n}_{\mathrm{m}}^{\beta}\right)$ which provides extremely fine-grained information about how electrons and their spins distribute in a molecule.

We have stressed [25] that all standard localization and delocalization descriptors used in real space methodologies are nothing but the (multivariate) cumulant moments of the EDF. The average population of a domain $\Omega_{i}$, the $N_{i}$ introduced above, is obviously equal to $N_{i}=\sum_{\left\{n_{p}\right\}} n_{i} \times p\left(\left\{n_{p}\right\}\right)=$ $\sum_{n_{i}} n_{i} p_{i}\left(n_{i}\right)$. In a similar way we can obtain its variance, skewness, etc. If the variance of the population of domain $\Omega_{i}, \operatorname{var}\left(n_{i}\right)=\sum_{i}\left(n_{i}-N_{i}\right)^{2} p\left(n_{i}\right)$ vanishes, then its electron population does not fluctuate, i.e. the electrons are localized. If it does, some of the electrons lying in $\Omega_{\mathrm{i}}$ may be found in other domains, i.e. they delocalize, and the covariance between $\Omega_{\mathrm{i}}$ and another domain, $\Omega_{\mathrm{j}}$, will not vanish: $\operatorname{cov}\left(\mathrm{n}_{\mathrm{i}}, \mathrm{n}_{\mathrm{j}}\right)=\sum_{\left\{\mathrm{n}_{\mathrm{p}}\right\}}\left(\mathrm{n}_{\mathrm{i}}-\mathrm{N}_{\mathrm{i}}\right)\left(\mathrm{n}_{\mathrm{j}}-\mathrm{N}_{\mathrm{j}}\right) \mathrm{p}\left(\left\{\mathrm{n}_{\mathrm{p}}\right\}\right) \neq 0$. The foundation of the statistical 
theory of the chemical bond lies in the deep relation between these moments (which are actually cumulant moments) and the traditional bonding descriptors. [25] For instance, the Wiberg-Mayer bond index $[26,27]$ between centers $\mathrm{i}$ and $\mathrm{j}, \delta^{\mathrm{ij}}=2 \sum_{\mu \in \mathrm{i}, \nu \in \mathrm{j}}(\mathrm{PS})_{\mu \nu}(\mathrm{PS})_{\nu \mu}$, which is constructed from atom-centered (i) primitive functions $(\mu)$ and widely used, is immediately generalized in real space to the two-center DI, which is nothing but the inter-domain covariance, $\mathrm{DI}(\mathrm{i}, \mathrm{j})=\delta^{\mathrm{ij}}=-2 \operatorname{cov}\left(\mathrm{n}_{\mathrm{i}}, \mathrm{n}_{\mathrm{j}}\right)$. It is customary to call the diagonal $\mathrm{i}=\mathrm{j}$ elements localization indices, $\mathrm{LI}(\mathrm{i})=\lambda^{\mathrm{i}}=\mathrm{N}_{\mathrm{i}}-\operatorname{var}\left(\mathrm{n}_{\mathrm{i}}\right)$, which provide effective measures of the number of electrons localized in domain $\Omega_{\mathrm{i}}$. Since the sum of variances and covariances vanishes (all population fluctuation has to go somewhere), a sum rule appears, and $\mathrm{N}=\sum_{\mathrm{i}} \lambda^{\mathrm{i}}+\sum_{\mathrm{i}>\mathrm{j}} \delta^{\mathrm{ij}}$. Using further order statistical moments of the EDF, multi-center fluctuation measures (multi-center delocalization indices) can also be defined. [16-18]

It is also possible to partition the DI into independent channels through a diagonalization involving a further order density that leads to a set of one-electron eigenfunctions called natural adaptive orbitals (NAdOs). Their occupation numbers add to $\delta^{\mathrm{ij}}$. [28] NAdOs provide an appealing partition of the bond order into terms which may be associated to bonding contributions.

In recent years, several rigorous bond-energy bond-order (BEBO) relations have been uncovered using real space descriptors. [29-31] It has been shown, for instance, that within the IQA framework the first order (dominant) electrostatic and covalent energy contributions to the interaction between atoms $\mathrm{i}$ and $\mathrm{j}$ can be written as $\mathrm{E}_{\mathrm{els}}^{\mathrm{ij}} \sim-\frac{\iota^{\mathrm{ij}}}{\mathrm{R}_{\mathrm{ij}}}, \quad \mathrm{E}_{\mathrm{cov}}^{\mathrm{ij}} \sim-\frac{1}{2} \frac{\delta^{\mathrm{ij}}}{\mathrm{R}_{\mathrm{ij}}}$, where $\iota^{\mathrm{ij}}=-\mathrm{Q}_{\mathrm{i}} \mathrm{Q}_{\mathrm{j}}$ is an ionic bond order defined from the atomic net charges. An appealing electrostatic model of chemical bonding can be built from these relations. [31]

The statistical link between the fluctuation of electron populations and the standard energetic and bond order descriptors used in real space paves the way to scanning fully the properties of bond indices through simple models. An example of how this can be done has already been presented. [21] In a two-center, two-electron (2c,2e) system there are only three RSRSs: $(2,0),(1,1),(0,2)$, where we label how many electrons lie in each domain. The EDF space is thus two-dimensional, since $\mathrm{p}(2,0)+\mathrm{p}(1,1)+\mathrm{p}(0,2)=1$, and all bond indices become fully mapped in this $2 \mathrm{D}$ space. A convenient coordinate system is constructed with the probability that any of the electrons lie in one of the basins, which we call $\mathrm{p}$ and provides a measure of heteropolarity, and a correlation factor $-1 \leq f \leq 1$ that determines how the electronic motion is correlated. $f=1$ means that an electron is completely excluded from one domain if the other is already in it (positive correlation) and 
$f=-1$ implies that the two electrons are always found together within the same domain (negative correlation). The correlation factor here defined plays the same role as that used in density matrix theory. The $(p, f)$ pair describes fully a $2 c, 2 e$ link at this coarse-grained level: $p(2,0)=p^{2}-p(1-p) f$, $p(1,1)=2 p(1-p)(1+f)$ and $p(0,2)=(1-p)^{2}-p(1-p) f$.

With this parametrization, the delocalization index becomes $\delta=4 p(1-p)(1-f)$. In standard weakly correlated bonds with positive $\mathrm{f} \approx 0$, the EDF becomes binomial, and $\delta$ peaks at $\delta=1$ for a purely covalent homopolar link with $\mathrm{p}=1 / 2$. As correlation, $\mathrm{f}$, or polarity, $\mathrm{p}$, increases, $\delta$ decreases. Moreover, for non-correlated links with $\mathrm{f}=0, \iota^{\mathrm{ij}}=1-\delta^{\mathrm{ij}}$ so, in agreement with standard wisdom, the ionic and covalent bond orders are inversely correlated.

Deviations to this canonical framework appear as $f$ strays significantly from zero. Strong positive correlation induces localization (decreases $\delta$ ) and this justifies that alleged multiply (up to quintuply or sextuply) bonded metal dimers [32] display much lower Dls than these naïve electron counts, for instance. Another virgin territory out there to explore lies in $f<0$ regions. As we will show here, they are actually found in ESs.

Negatively correlated bonds imply a bosonization of the electron system: electrons do not avoid each other, but on the contrary try to delocalize together. In our example, the most extreme $2 c, 2 e$ case with $\delta=2$ occurs when $\mathrm{p}(0,2)=\mathrm{p}(2,0)=1 / 2$ and $\mathrm{p}(1,1)=0$, i.e. when there is a resonance between the two valence bond ionic structures. Notice that we will be using the label ionic in two different senses: valence bond ionic resonance and charge transfer ionicity. In the first sense there is no overall charge transfer.

As allowing $f$ to become negative may bring new bonding situations to the fore, increasing the number of centers expands the bonding landscape considerably. Besides permitting many-center electron delocalization, multicenter bonding is a discipline on its own, the presence of a third center introduces the possibility of negative DIs. In diatomics this is not possible: $\operatorname{cov}\left(n_{i}, n_{j}\right)$ is negative semidefinite, for increasing the population of one center implies decreasing it on the other thanks to particle number conservation. This is no longer true when a third atom appears, and weird fluctuations may arise in which increasing the population of a center (positive fluctuation) is also accompanied by an increase in the number of electrons of the other. This implies some type of concerted electronic dance involving the third center. We will also report this new behavior here.

Within the EDF formalism, each RSRS $\left\{n_{p}\right\}$ has a distinct contribution to any given bonding 
descriptor like $\delta^{\mathrm{ij}}: \delta^{\mathrm{ij}}\left(\left\{\mathrm{n}_{\mathrm{p}}\right\}\right)=-2\left(\mathrm{n}_{\mathrm{i}}-\mathrm{N}_{\mathrm{i}}\right)\left(\mathrm{n}_{\mathrm{j}}-\mathrm{N}_{\mathrm{j}}\right)$. This contribution is weighted by the probability of the RSRS to give the final DI, $\delta^{i j}=\sum_{\left\{n_{p}\right\}} \delta^{i j}\left(\left\{n_{p}\right\}\right) p\left(\left\{n_{p}\right\}\right)$. In this sense, a RSRS may be understood to favor or oppose the final average bonding descriptor. In a homodiatomic with centers $\mathrm{a}, \mathrm{b}$, for instance, the neutral RSRS with equal number of electrons in each atomic domain $\left(\mathrm{n}_{\mathrm{a}}=\right.$ $\left.\mathrm{N}_{\mathrm{a}}=\mathrm{n}_{\mathrm{b}}=\mathrm{N}_{\mathrm{b}}=\mathrm{N} / 2\right)$ ) does not contribute to $\delta^{\mathrm{ab}}$. Only non-neutral structures in which electrons are delocalized add to the DI. Similarly, for a heterodiatomic in the charge transfer (CT) ionic limit, e.g. a LiF molecule in which the average atomic populations are close to $\mathrm{Li}^{+}$and $\mathrm{F}^{-}$, the fully CT ionic RSRS will not contribute appreciably to $\delta$, and only those in which electrons are transferred back to Li will do.

\section{The Hubbard model in molecules}

The Hubbard model [22] was proposed in solid state physics as possibly the simplest lattice model able to describe the metal-insulator transition. This is done through a modulation of the extent of electron delocalization, achieved through an energetic penalty parameter $U$ that difficulties electrons from occupying the same lattice site. Despite its simplicity, analytical solutions are only known in the one-dimensional case, [33] and thanks to the rich physics it embodies, it has been thoroughly used and generalized in condensed matter physics.

In the standard Hubbard Hamiltonian each lattice site i can host up to two opposite spin electrons (in a single effective orbital). At half-filling, i.e. when there are as many electrons as sites, electrons can hop between (opposite spin) singly occupied nearest neighbors. The hopping ability is controlled via a site coupling parameter $\mathrm{t}$. In second quantized form, $\mathrm{H}=-\mathrm{t} \sum_{\langle i, j\rangle, \sigma}\left(\mathrm{c}_{\mathrm{i} \sigma}^{+} \mathrm{c}_{\mathrm{j} \sigma}+\mathrm{c}_{\mathrm{j} \sigma}^{+} \mathrm{c}_{\mathrm{i} \sigma}\right)+\mathrm{U} \sum_{\mathrm{i}} \mathrm{n}_{\mathrm{i} \uparrow} \mathrm{n}_{\mathrm{i} \downarrow}$, $\mathrm{c}_{\mathrm{i} \sigma}$ creates $\sigma$-spin electron at site $\mathrm{i}, \mathrm{n}_{\mathrm{i} \uparrow}, \mathrm{n}_{\mathrm{i} \downarrow}$ are spin-resolved electron number operators, and the $\langle i, j\rangle$ sum contains only nearest neighboring $i, j$ sites. It is usual that $U$, the on-site repulsion, be a semipositive definite parameter. This is the repulsive Hubbard lattice, where $r=U / t$ acts as a dimensionless correlation strength. At $U=0$ the model collapses onto the tight-binding or Hückel approximation, known to all chemists, whereas as $U$ tends to infinity the electrons fully localize at their sites. We have already noticed [34] that the standard order parameter used to locate a possible metal-insulator transition, $\mathcal{D}=\left\langle n_{\mathrm{i} \uparrow} n_{\mathrm{i} \downarrow}\right\rangle$, is nothing but $\mathrm{p}(2)$, the probability of double occupancy of a site within the EDF formalism, and that a two sites Hubbard system has been successfully used 
as an analogue for all the singlet and triplet $\mathrm{Cl}$ states of dihydrogen in a minimal basis. In this way, the tools of the theory of chemical bonding in real space can be explored in other realms, offering valuable information about how strong Coulomb correlation induces electron localization.

Although less known, $U$ can be made negative, in what it is known as the attractive Hubbard model. [35] Now there is an energetic reward for electrons to pair up at sites, leading to a bosonization of the electron system. It is rather clear that the sign and magnitude of $U$, or of $r$, are intimately related to the correlation factor $f$ defined above, and that the Hubbard model contains all the ingredients needed to simulate $2 \mathrm{c}, 2 \mathrm{e}$ bonds and to mimic the behavior of more realistic systems.

\section{Computational Details}

To provide convincing proofs of our claim that most, if not all, of the weird statistical electron distributions that can be envisioned from EDFs can actually be found in excited states, we have performed model (Hubbard) and high level calculations in simple systems, some of them from our previous work focused on energy transfer. [11]

Calculations were done as follow. The Hubbard model wavefunctions were obtained using the PySCF [36] suite. Lattice-coarse density matrices were extracted and analyzed. For the electronic structure calculations we have employed the full configuration interaction $(\mathrm{FCl})$ and multi-reference configuration interaction with single and double excitations (MRCI-SD) levels of theory. Details on the $\mathrm{MRCl}-\mathrm{SD}$ computations can be found in a previous article. [11] Briefly, the reference state was constructed as an equal weight state averaged wavefunction (SA-CASSCF) with the d-aug-cc-pVDZ [37] basis set. The active spaces are (2 electrons, 10 orbitals) in the case of $\mathrm{H}_{2},(4,4)$ for $\mathrm{He}_{2},(4,6)$ for $\mathrm{LiH},(6,6)$ for $\mathrm{LiF}$ and $(2,2)$ for $\mathrm{C}_{2} \mathrm{H}_{4}$. For ethylene, the minimum energy path (starting from the Franck-Condon region) for the first valence excited state was obtained at the XMS-CASPT2/ccpvdZ level, and subsequently, single point $\mathrm{MRCl}$ calculations were carried out. In the $\mathrm{H}_{4}$ system $\mathrm{FCl}$ calculations were done in the $A_{g}$ representation of the point group $D_{2 h}$ with the aug-cc-pvdz. [37] The MRCI-SD and XMS-CASPT2 electronic structure calculations were respectively performed using Molpro [38] and BAGEL; [39] PySCF [36] was used for the FCI level.

The density matrices provided by the electronic structure codes were fed into the PROMOLDEN [40] code to obtain atomic overlap matrices (with the lattice or QTAIM partition). Electron distribution 
functions were obtained with our in-house code EDF [23] and natural adaptive orbitals (NAdOs) with DENMAT, [41] another in-house code.

\section{Results and Discussion}

We devote this section to comment on both model and actual electronic structure calculations in a few exemplifying systems. We refer the reader to the supplementary material (ESI) for comprehensive Tables, as well as for a simple comparison with standard molecular orbital results, particularly as natural occupation numbers are regarded. We will start by discussing several dihydrogen states together with the two-sites Hubbard model at half filling. This will serve us to examine the chemical space of a homodiatomic $2 c, 2 e$ interaction. We will then shift to heterodiatomics, studying charge transfer processes induced by avoided crosssings. Negative delocalization indices will be found in an interesting exploration of the $\mathrm{D}_{4 \mathrm{~h}}$ states of $\mathrm{H}_{4}$ and, finally, a less academic example of conical intersection in ethylene will be presented.

\section{The 2c,2e homodiatomic case: $\mathrm{H}_{2}$ and the two-sites Hubbard solution.}

Following our previous work, [11] EDFs and bonding descriptors across the potential energy surfaces (PES) of the $\mathrm{X}^{1} \Sigma_{\mathrm{g}}^{+}(\mathrm{S} 0)$, the $\mathrm{B}^{1} \Sigma_{\mathrm{u}}^{+}(\mathrm{S} 1)$, the double minimum $\mathrm{E}, \mathrm{F}^{1} \Sigma_{\mathrm{g}}^{+}(\mathrm{S} 2)$ and the $\mathrm{b}^{3} \Sigma_{\mathrm{u}}^{+}(\mathrm{T} 1)$ states of $\mathrm{H}_{2}$ have been examined. S0 and T1 have been extensively analyzed before, $[42,43]$ but are included for completeness. Although all the $B^{1}$ and $E, F$ states dissociate to $H(1 s)+H(2 I)$, only the $\mathrm{E}$ state is directly correlated to this limit, while the $\mathrm{B}^{1}$ state and the $\mathrm{F}$ minimum correlate to the $\mathrm{H}^{+}+\mathrm{H}^{-}$and are thus considered to have a clearly valence bond ionic character. This was clearly found from our IQA energetic point of view. [11] It is this valence bond ionic or zwitterionic character the one we are interested now. At the $\mathrm{H}^{+}+\mathrm{H}^{-}$dissociation limit, which is not attained due to several avoided crossings, the two electrons are either found on the left or right domains, so that $\mathrm{p}(2,0)=\mathrm{p}(0,2)=1 / 2, \mathrm{p}(1,1)=0$, and $\delta^{\mathrm{ij}}=-2 \operatorname{cov}\left(\mathrm{n}_{\mathrm{i}}, \mathrm{n}_{\mathrm{j}}\right)$, the DI equals 2.0, doubling the limit for normal 2c,2e bonds, which is one. In a normal link with positive (or null) correlation factor $\mathrm{f}$, the maximum value for $\delta=4 p(1-p)(1-f)$ is attained when $p=1 / 2, f=0$.

Fig. 1 shows the LI and DI values for the ground state two-sites Hubbard solution at positive and 
negative $U$ values. The triplet state is not shown since double occupancy is forbidden in this case, so that its DI vanishes. Notice that being $\mathrm{p}=1 / 2, \mathrm{f}=1-\delta$, and $\mathrm{p}(2,0)+\mathrm{p}(0,2)=\delta / 2$, so that the Figure contains information of all these quantities at the same time. The repulsive $\mathrm{U} / \mathrm{t}$ region is well known. At the $U=0$ tight binding limit the solution is a single determinant, equivalent from the delocalization point of view to the Hartree-Fock model, $\delta=1$, the correlation factor $\mathrm{f}$ vanishes, and the two electrons are statistically independent. This gives rise to a symmetric binomial probability distribution, i.e. $\mathrm{p}(2,0)=\mathrm{p}(0,2)=1 / 4, \mathrm{p}(1,1)=1 / 2$. As $\mathrm{U} / \mathrm{t}$ tends to $+\infty$ and the on-site repulsion dominates, the electrons localize, $f \rightarrow 1$ and the $D I$ vanishes.

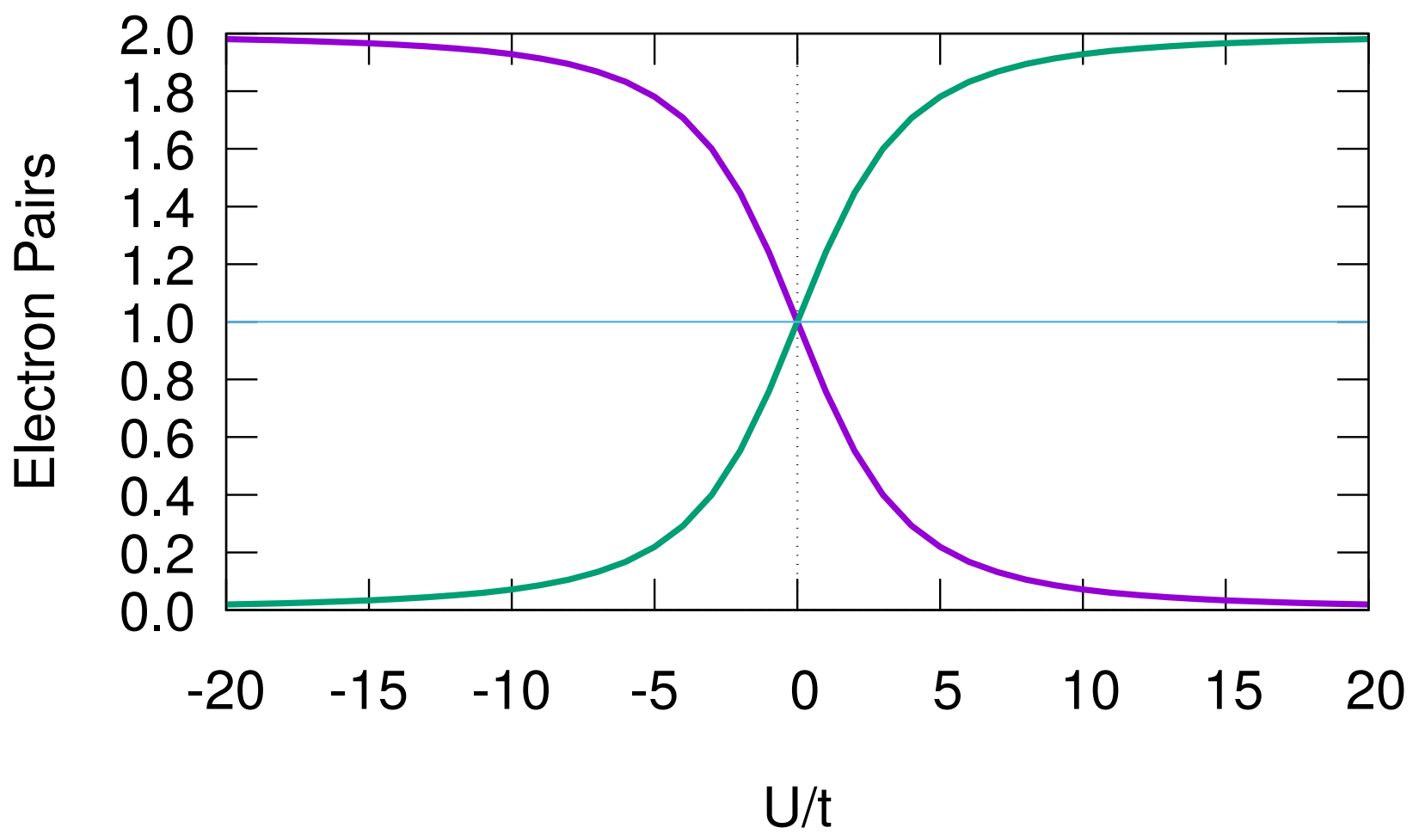

Figure 1: Localization ( $2 \lambda$, green) and delocalization ( $\delta$, purple) indices for the half filling two-sites Hubbard model. The localization index has been multiplied by a factor of two, so that $2 \lambda+\delta=\mathrm{N}=2$ at any $\mathrm{U} / \mathrm{t}$.

More interesting are the attractive Hubbard results. At negative $U$, where on-site double occupancy is incentivized, the LI and DI curves exchange roles with respect to those in the repulsive model. As $\mathrm{U} / \mathrm{t}$ tends to $-\infty$ the electrons pair-up, the valence bond resonance dominates so that $\mathrm{p}(1,1)$ and the $\mathrm{LI}$ vanish, and the variance of the EDF takes a maximum value. Notice that in this resonance bond, $\delta=2$ for a single pair of electrons, so that the generalization of the bond order concept by means of Dls includes these new situations naturally. The two curves in the Figure inter- 
sect at $\mathrm{U} / \mathrm{t}=0$, where the total weight of the ionic resonance (50\%) equals that of the ionic covalent one.

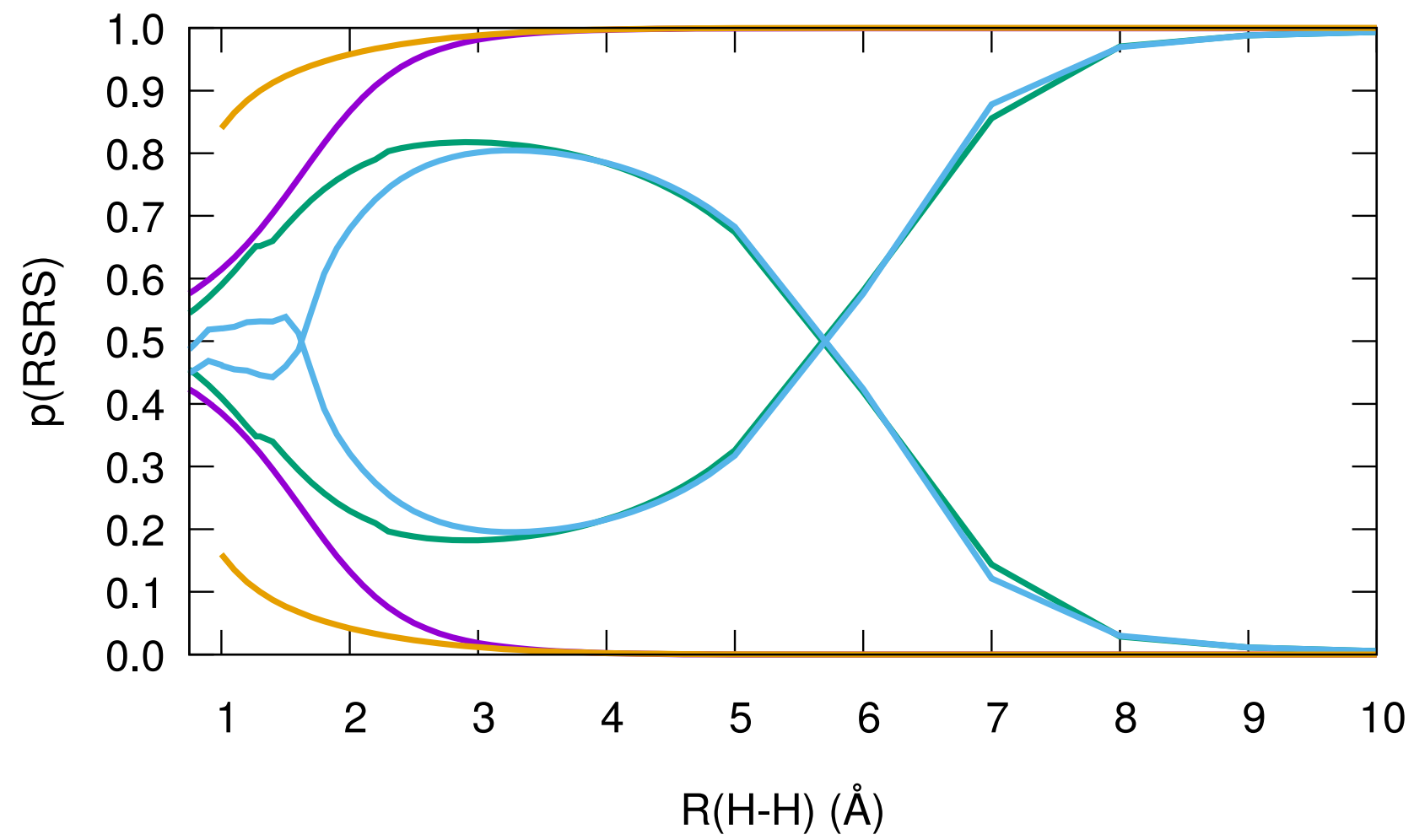

Figure 2: Evolution of the probability of each RSRS for the different states of $\mathrm{H}_{2}$ with distance. For convenience, the structure $\mathrm{p}(2,0)$ is multiplied by a 2 factor $(\mathrm{p}(2,0)+\mathrm{p}(0,2))$. Structures are identified by their dissociation limit: the $(1,1)$ tends to 1 , while the $(2,0)$ tends to 0 . States are distinguished by color: S0 in purple, S1 and S2 in green and blue, respectively, and T1 in orange.

Fig. 2 shows results from the MRCI-SD calculations in the $\mathrm{H}_{2}$ states analyzed in this work. The $\mathrm{DI}$ in this homodiatomic case is simply $\delta^{\mathrm{ij}}=-2[2 \mathrm{p}(2,0)(0-1)(2-1)]=4 \mathrm{p}(2,0)$. As stated, the behavior of the DI in the S0 and T1 states is well known, the former decaying sigmoidally while the latter goes to zero exponentially. This property is useful in the classification of bonded and non-bonded interactions. [44]

The first relevant difference between the S0 or T1 states and the excited singlets $\mathrm{S} 1$ and $\mathrm{S} 2$ is the much longer range of the interactions in the latter, in agreement with our previous results. The EDFs of both the S1 and S2 states are almost indistinguishable from dissociation down to about $3 \AA$. At very large distances both behave as normal states, decaying to neutral distributions where $\mathrm{p}(1,1)=1$. As the atoms approach each other they interact very strongly, and at a considerably large internuclear distance, about $5.7 \AA$, the $p(0,2)+p(2,0)$ curve crosses the $p(1,1)$ in the two 
states. The $\mathrm{B}^{1} \Sigma_{\mathrm{u}}^{+} \mathrm{S} 1$ state persists, from that distance to the shortest one used in our simulations, in an anomalous distribution with a total ionic resonance weight $(p(0,2)+p(2,0))$ larger than $50 \%$ and a DI greater than one. Even at its energy minimum, located at $=1.27 \AA, \delta=1.30$. We stress how easily the inversion points determining whether zwitterionic or normal states predominate are located using EDFs. The E, $F^{1} \Sigma_{g}^{+}$is resonance ionic from 5.7 to about $1.7 \AA$, where its behavior turns back to normal. Since the F energy minimum occurs at $2.32 \AA$, while the $E$ one is located at $\mathrm{R}=1.01 \AA$, the $\mathrm{E}$ electronic distribution is not zwitterionic, in agreement with common wisdom. The Dls at the E,F minima are 0.92 and 1.50 , respectively.

The $\mathrm{H}_{2}$ case just examined shows without doubt that Dls greater than one for a 2c,2e case are certainly possible. In this simple case a chemist would immediately associate them to ionic resonances. From a more physicist-like perspective they would be mapped to an attractive Hubbard model, a scenario related to the bosonization of pairs of electrons. We think that these real space descriptors may be important in developing chemical insight in fields like superconductivity or in providing independent tests of the nature of purportedly new types of bonding, like the charge-shift bond. $[45,46]$

\section{$\mathrm{He}_{2}$}

The lowest lying excited states of $\mathrm{He}_{2}$ have been studied thoroughly over the years. $[47,48]$ We have considered the lowest lying excimer singlets, $\left.S 1\left(A^{1} \Sigma_{u}^{+}\right)\right)$and $S 2\left(C^{1} \Sigma_{g}^{+}\right)$, that dissociate to $\mathrm{He}\left(1^{1} S_{0}\right)+\mathrm{He}\left(2^{1} S_{0}\right)$, and the first triplet, $T 1\left(A^{3} \Sigma_{u}^{+}\right)$, that dissociates to $\mathrm{He}\left(1^{1} S_{0}\right)+\mathrm{He}\left(1^{3} S_{1}\right)$. These three states display deep energy minima at $1.047,1.099$, ad $1.052 \AA$, respectively. As we showed in our previous report, [11] the IQA energetic point of view readily uncovers the entangled nature of the resonances at dissociation as well as the strong covalent bonding as measured by exchangecorrelation energies $\mathrm{V}_{\mathrm{xc}}^{\mathrm{AB}}$ in excess of $100 \mathrm{kcal} / \mathrm{mol}$.

Fig. 3 contains the EDFs of all the states. At all but the smallest distances only the $(2,2),(3,1)$ and $(1,3)$ structures are populated significantly. As in the S1 and S2 excited states of dihydrogen, the interaction between the two atoms is very long ranged, and the EDF deviates noticeably from the $(2,2)$ dissociation limit even at $5 \AA$. Both $S 1$ and S2, which converge at dissociation, have indistinguishable distributions (and energies) at distances larger than $3.5 \AA$, where they start to 


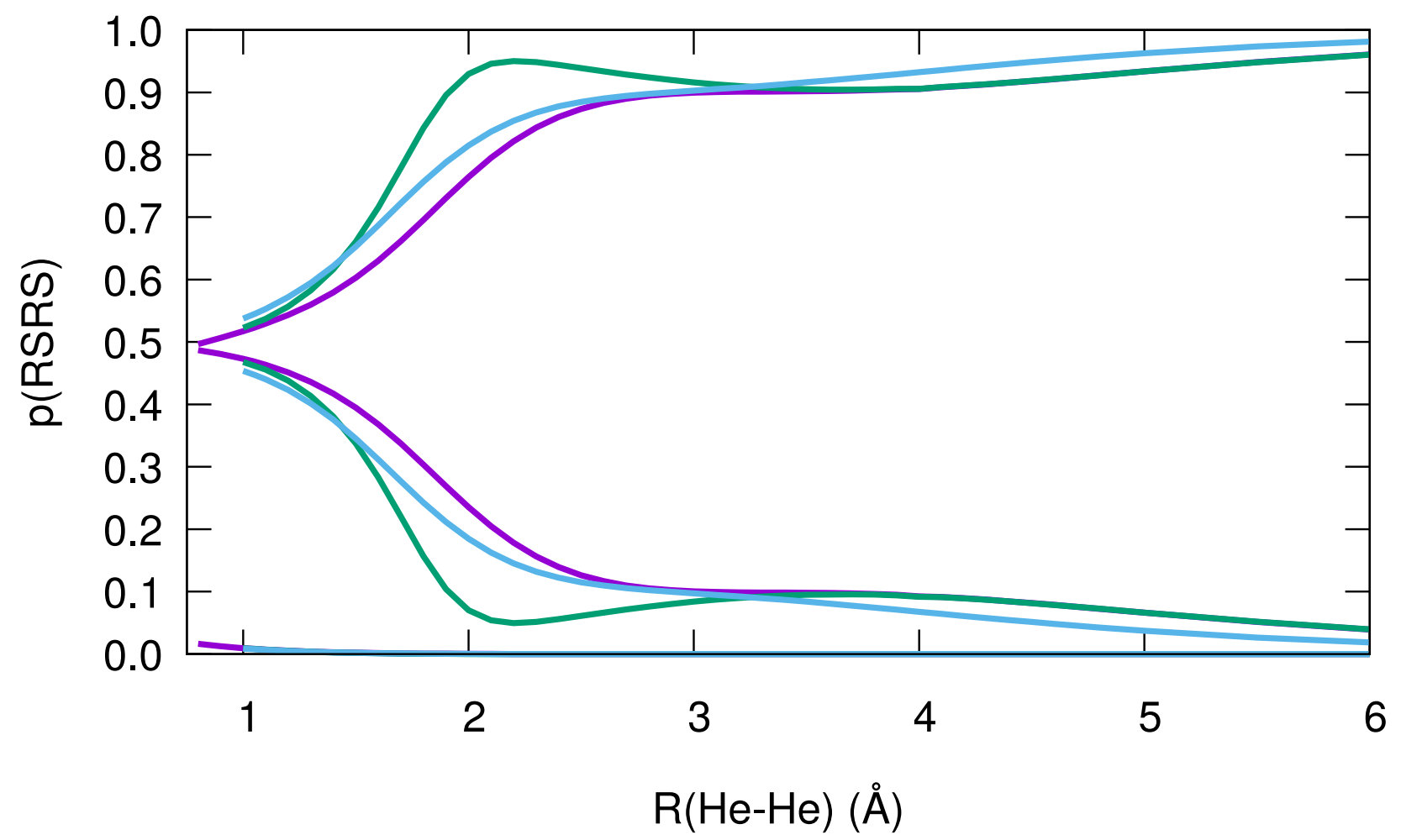

Figure 3: Evolution of the EDF in the excimer states of $\mathrm{He}_{2}$. The probabilities of the $(3,1)$ and $(4,0)$ RSRSs have been multiplied by two, accounting for the total $(3,1)+(1,3)$ or $(4,0)+(0,4)$ components of the distribution. Structures are identified by their limiting behavior: $(2,2)$ tends to 1 , while $(3,1)$ tends to 0 . The $(4,0)$ structure remains negligible except at very low distances. States are distinguished by color: $\mathrm{S} 1$ in purple, $\mathrm{S} 2$ in green and $\mathrm{T} 1$ in blue.

deviate. Several intra-atomic rearrangements take place at smaller distances which are not reflected on the interatomic interactions. S2 shows a minimum in the probability of the $(3,1),(1,3)$ RSRSs at about $2.2 \AA$, distance at which a maximum in its potential energy curve is found. As we see from the EDF, this is due to inhibited delocalization, very likely due to its interaction with another state.

If the $(4,0),(0,4)$ structures are neglected, the EDFs of the three states fall at short distances onto the binomial distribution corresponding to two delocalized electrons, as in $\mathrm{H}_{2}$. In fact, the Dls of the S1, S2, and T1 states at their equilibrium configurations are 1.00,0.97, and 0.96, respectively, a bond order equal to one. However, this distribution should actually be read as that coming from two 2c,1e bonds. We think that all standard bonds should be understood in terms of nc, 1e contributions, an idea that will be pursued elsewhere. Briefly, a Lewis pair is usually the result of the Fermi hole around one electron leaving room so that an opposite spin partner can occupy it. The two electrons seem to dance together, although they are basically independent statistically. A typical $2 c, 2 e$ link is thus better seen as two independent 2c,1e bonds. In the present case two different delocalization 
channels open when one of the atoms is excited, as shown in the left panel of Fig. 4. These lead to the exchange of the excitation from one atom to the other, each of them contributes approximately 0.50 to the total DI in any of the states. The two main NAdOs in the T state, found on the right panel, are seen to be $\sigma_{\mathrm{g}, 1 \mathrm{~s}^{-}}$and $\sigma_{\mathrm{g}, 2 \mathrm{~s}}$-like, as expected, contributing to the DI 0.476 and 0.437 , respectively. A similar situation is found in the two singlets.

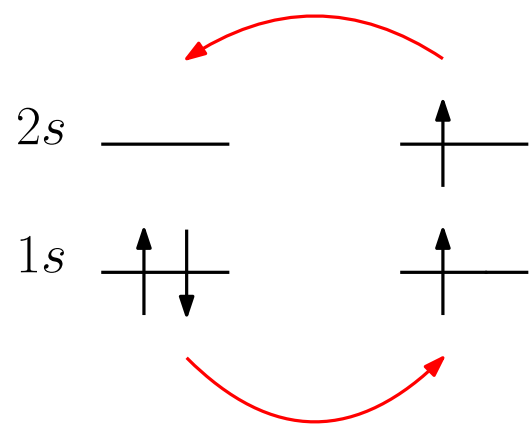

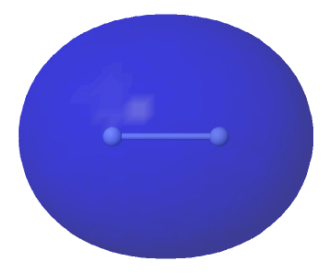

0.476

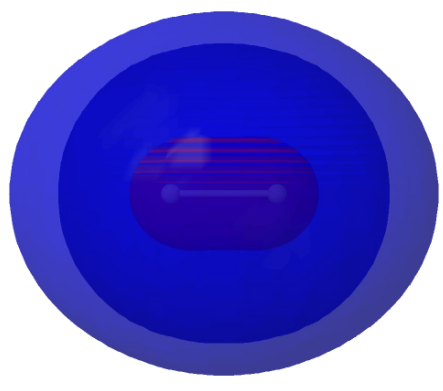

0.437

Figure 4: The two two-center, one-electron bonds in the $\mathrm{T} 1$ state of $\mathrm{He}_{2}$ at its equilibrium distance. Left, scheme showing the two independent delocalization channels. Right, $|\phi|=0.036$ au isosurfaces of the two main NAdOs of the system. The contributions of each NAdO to the DI is also shown.

\section{The 2c,1e charge transfer limit: LiF and LiH}

We have examined the $1^{1} \Sigma^{+}$and $2^{1} \Sigma^{+}$states of $\mathrm{LiF}$ and $\mathrm{LiH}$. The formation of the LiF molecule involves an avoided crossing ocurring at about $R=6.64 \AA$ coupled with an electron transfer of 0.94 e towards the $\mathrm{F}$ atom. At dissociation the $\mathrm{S} 0$ and $\mathrm{S} 1$ states tend to neutral atoms and to the $\mathrm{Li}^{+}, \mathrm{F}^{-}$ pair, respectively, and are known as the covalent and ionic states. They are interconverted at the avoided crossing, in a transition that occurs suddenly, within a very narrow distance window. The equilibrium distance of the ground state is located at $R_{\text {eq }}=1.6 \AA$.

The evolution of the EDF is plotted in Fig. 5. There are only two RSRSs contributing nonnegligibly to the EDF except at small distances: the neutral $(3,9)$ and the charge transfer ionic $(2,10)$ ones. We find absolutely remarkable that the avoided crossing can be neatly located from the contact point of the neutral and ionic states at $p=1 / 2$. Notice that both charge resonances, as exemplified by $\mathrm{H}_{2}$, and charge transfers can be equally found. Even more interesting is the fact that from the dissociation limit to $\mathrm{R} \approx 5 \AA$ the S0 and S1 EDFs are almost exact mirror images. 
The $(2,10)$ component in one state is equal to the $(3,9)$ component in the other and viceversa. This means that whatever happens in the ground state is mirrored in the excited state, and that as charge transfer starts in one direction in S0, exactly the same charge transfer, in the opposite direction, is found in $\mathrm{S} 1$. One and the same parameter, e.g. $\mathrm{Q}(\mathrm{Li})$, controls the evolution of both states. Only for distances smaller than $5 \AA$ do the two states start to diverge. We stress that the EDF behavior is correlated to standard molecular orbital indicators although, in our opinion, the EDF offers a much richer view of chemical processes. For instance, we include in the ESI the evolution of the natural orbital occupancies for this and some other of our examples.

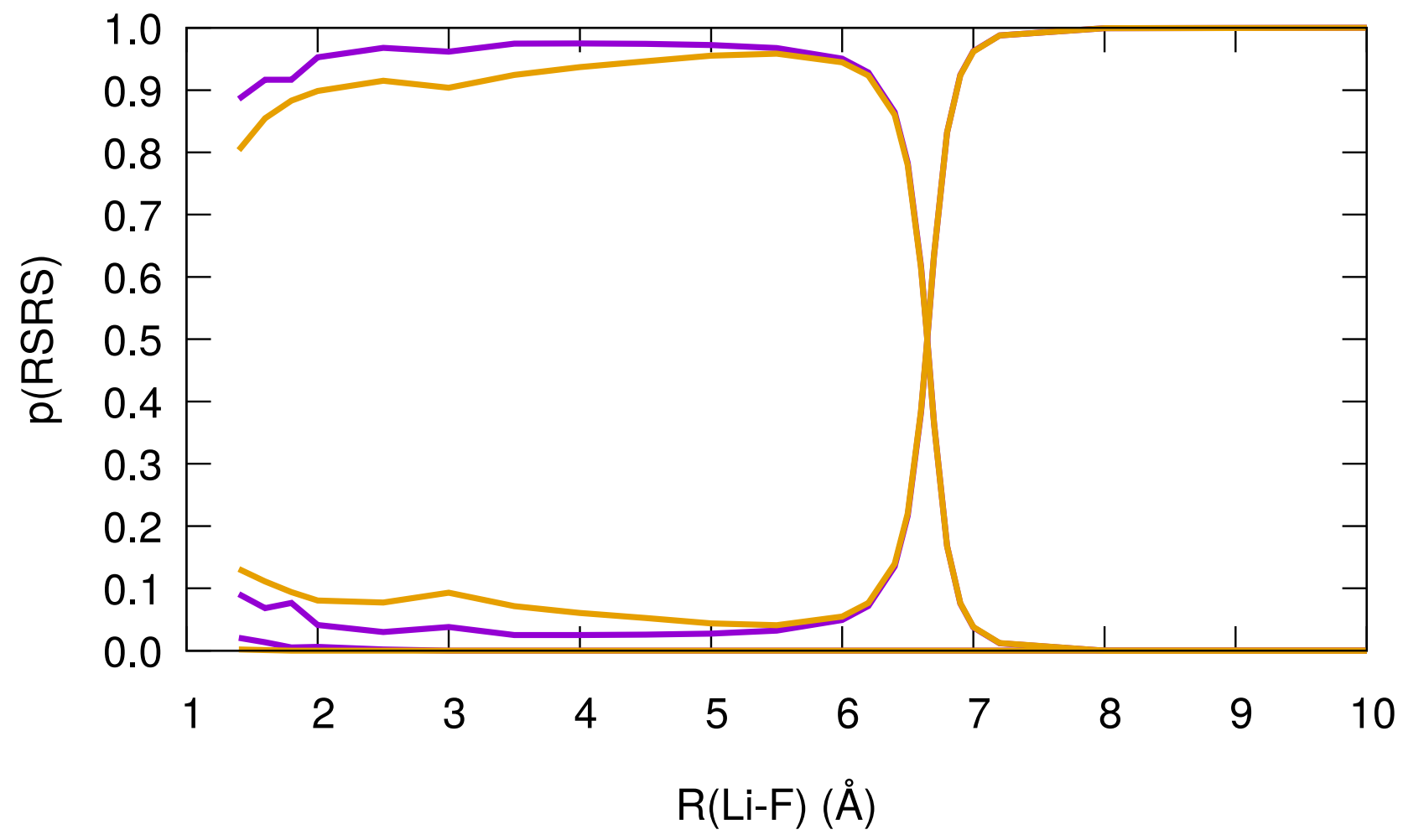

Figure 5: Evolution of the EDF in LiF. The left population is that of the Li atom. Structures are identified easily: $p(2,10)$ for S0 and $p(3,9)$ for $S 1$ tend to 0 at large distance, while $p(3,9)$ for S0 and $\mathrm{p}(2,10)$ for $\mathrm{S} 1$ tend tend to 1 in the same limit. $\mathrm{p}(1,11)$ remains negligible in both states except at the lowest distances. States are distinguished by color: S0 in purple and S1 in orange.

The one-parameter one-electron transfer nature of the ionic-covalent transition in this system is clearly evidenced in Fig. 6, where the DI of both states is plotted against the LiF internuclear distance. It is again remarkable that $\delta^{\mathrm{LiF}}$ for the S0 and S1 states are hard to distinguish visually in a wide region around the avoided crossing point, in which the DIs of both states are equal and equal to $1 / 2$. We thus have two states with the same delocalization behavior. 
A simple 2c,1e model sheds light on why this is so. Since only the $(2,10)$ and $(3,11)$ RSRSs are important, we may envision an electron flowing from the Li atom toward the F moiety in the S0 state as we approach the crossing from dissociation and an electron flowing from $\mathrm{F}^{-}$to $\mathrm{Li}^{+}$in the $\mathrm{S} 1$ state. If the probability that this mobile electron is found in the $\mathrm{Li}$ atom is called $\pi$, then $\mathrm{p}(2,10)=1-\pi$ and $\mathrm{p}(3,11)=\pi$ for S0, while $\mathrm{p}(2,10)=\pi$ and $\mathrm{p}(3,11)=1-\pi$ for S1. This provides $\delta=2 \pi(1-\pi)$ in both cases. Given that $\pi$ (or $1-\pi$ ) can also be interpreted as the charge transfer, the model provides a quadratic coupling between $\mathrm{Q}$ and $\delta$.

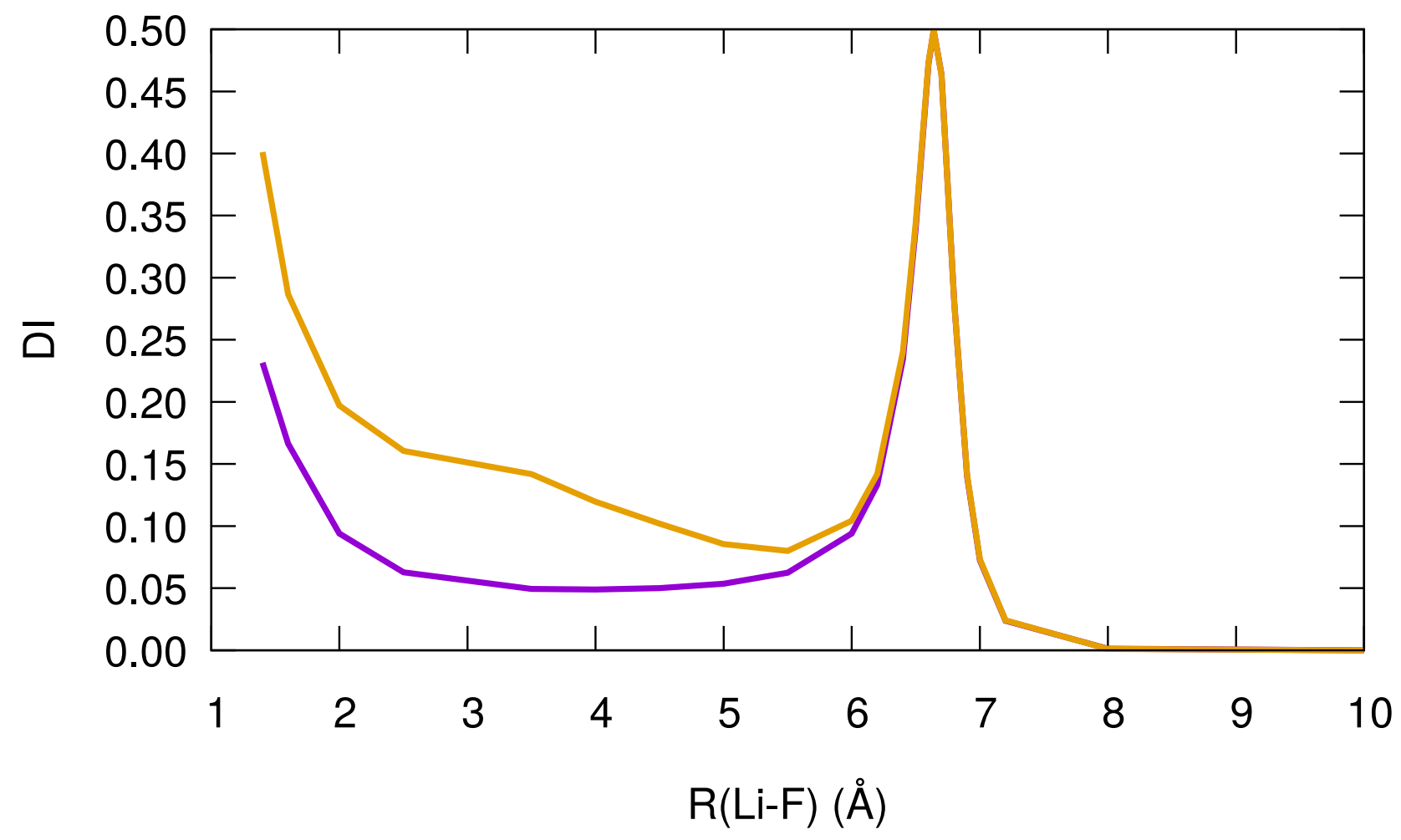

Figure 6: Evolution of the delocalization index in LiF. The S0 and S1 states are plotted in purple and orange, respectively.

The $\mathrm{LiH}$ case is similar, but includes a number of complications stemming from the low electron affinity of $\mathrm{H}$, which gives rise to a sequence of avoided crossings that impede the dissociation of the $S 1$ state to the ionic limit. Actually, a full configuration interaction calculation on the first four ${ }^{1} \Sigma$ roots (see Fig. S1) shows that the Coulombic tail of the ionic $\mathrm{Li}^{+}-\mathrm{H}^{-}$state crosses with S3 (which dissociates to $1 s^{2} 3 p^{1}-{ }^{2} \mathrm{P}^{0} \mathrm{Li}$ and $1 \mathrm{~s}-{ }^{2} \mathrm{~S} \mathrm{H}$ ), S2 (dissociating to $1 \mathrm{~s}^{2} 3 \mathrm{~s}^{1}-{ }^{2} \mathrm{~S} \mathrm{Li}$ and a $1 \mathrm{~s}-{ }^{2} \mathrm{~S} \mathrm{H}$ ) S1 (evolving to $1 \mathrm{~s}^{2} 2 \mathrm{p}^{1}-{ }^{2} \mathrm{P}^{0} \mathrm{Li}$ and $1 \mathrm{~s}-{ }^{2} \mathrm{~S} \mathrm{H}$ ) and S0. The last covalent-ionic avoided crossing occurs at a considerably lower internuclear distances (in our computation, at about $3.4 \AA$ ) than in LiF, so that the performance of the one-parameter model worsens. Fig. 7 shows the EDF of the S0 
and $\mathrm{S} 1$ states from our MRCI-SD calculation, which displays a good agreement with the $\mathrm{FCl}$ one for these two states, with one and two avoided crossings, respectively. Now a very clear crossing in So is found between 3 and $4 \AA$ that takes the the $(2,2)$ RSRS to fully dominate at shorter distances, confirming the charge-transfer ionic nature of the $\mathrm{LiH}$ ground state. This is close to, although not coincident with the EDF crossing in the S1 state, at about $3.7 \AA$. However, if we increase R, a maximum (minimum) in the $(2,2)((3,1))$ RSRSs develops that leads to a second crossing at 5.1 $\AA$. The valence state of the Li moiety in the $\mathrm{S} 1$ state thus evolve from $2 \mathrm{~s}^{1}$ at short distances, to $2 \mathrm{~s}^{0}$ (ionic) at intermediate range to $2 \mathrm{p}^{1}$ at dissociation. This can be fully corroborated through a NAdOs analysis, which will not be shown here.

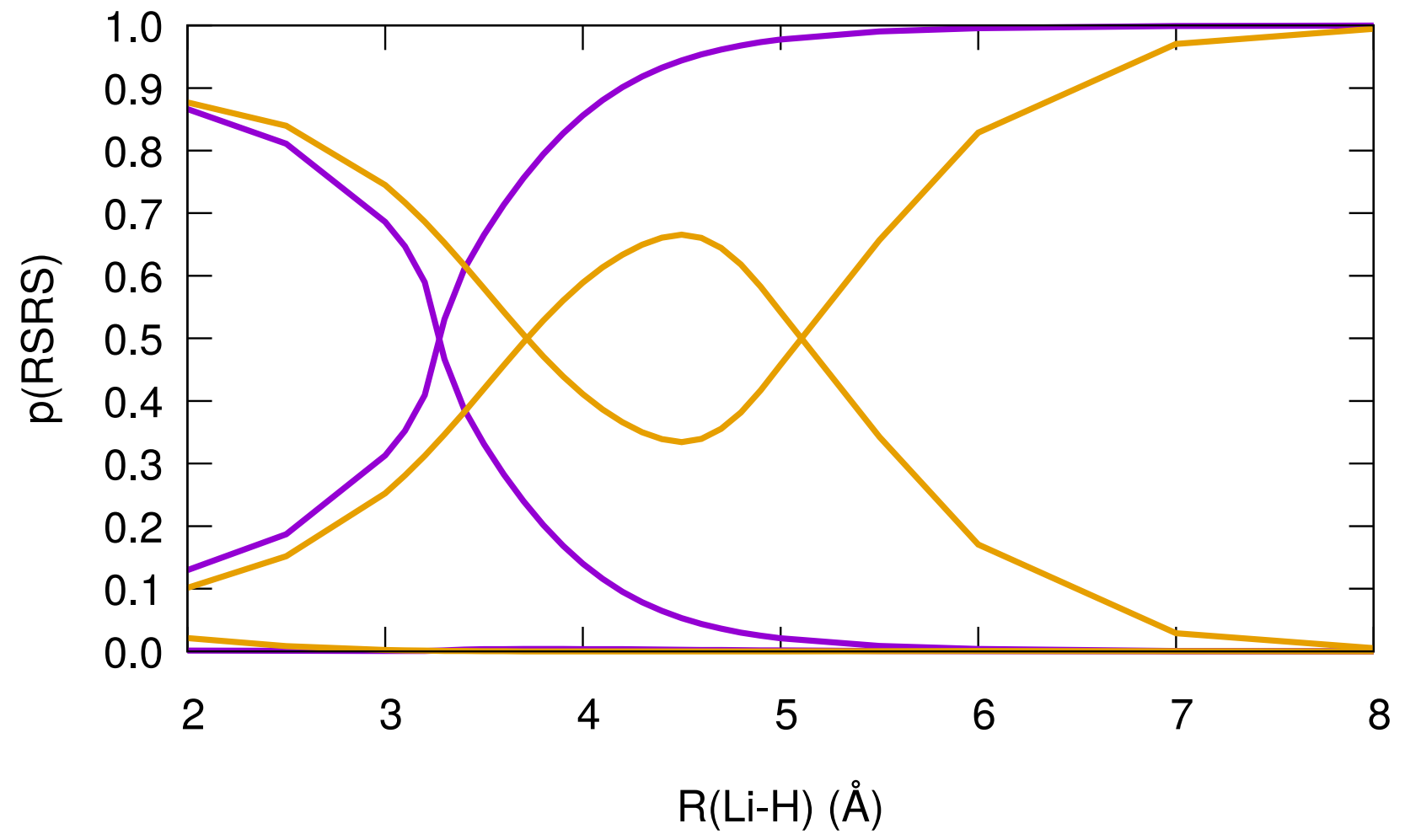

Figure 7: Probability of each RSRS for the S0/S1 states of LiH. The S0/S1 probabilities are shown in purple/orange. The probability of the $(4,0) \mathrm{RSRS}$ is negligible except at very low distances. In both states, the probability of the $(3,1)$ and $(2,2)$ RSRSs tend to 1 and 0 at dissociation, respectively.

\section{Expanding the landscape: $\mathrm{H}_{4}$ and the four-sites Hubbard model.}

We will now examine the influence that the existence of more than two centers has on the statistics of the electron distribution. We will avoid the possibly simplest system, $\mathrm{H}_{3}$, since several other factors, particularly the occurrence of conical intersections, contribute to blur the overall image. We 
turn then to a symmetric moiety with four sites, which will be examined both at the Hubbard model level and after actual electronic calculations in the $\mathrm{D}_{4 \mathrm{~h}}$ geometry of $\mathrm{H}_{4}$.

Fig. 8 contains a scheme with the atomic labelling used together with the non-equivalent RSRSs of $\mathrm{H}_{4}$. Only Hubbard compatible structures are shown with maximum doubly occupied sites. This restriction is no longer true in the $\mathrm{H}_{4}$ CAS calculations, but all RSRSs with triply or quadruply occupied $\mathrm{H}$ atoms have very low, negligible probabilities, and will not be considered.
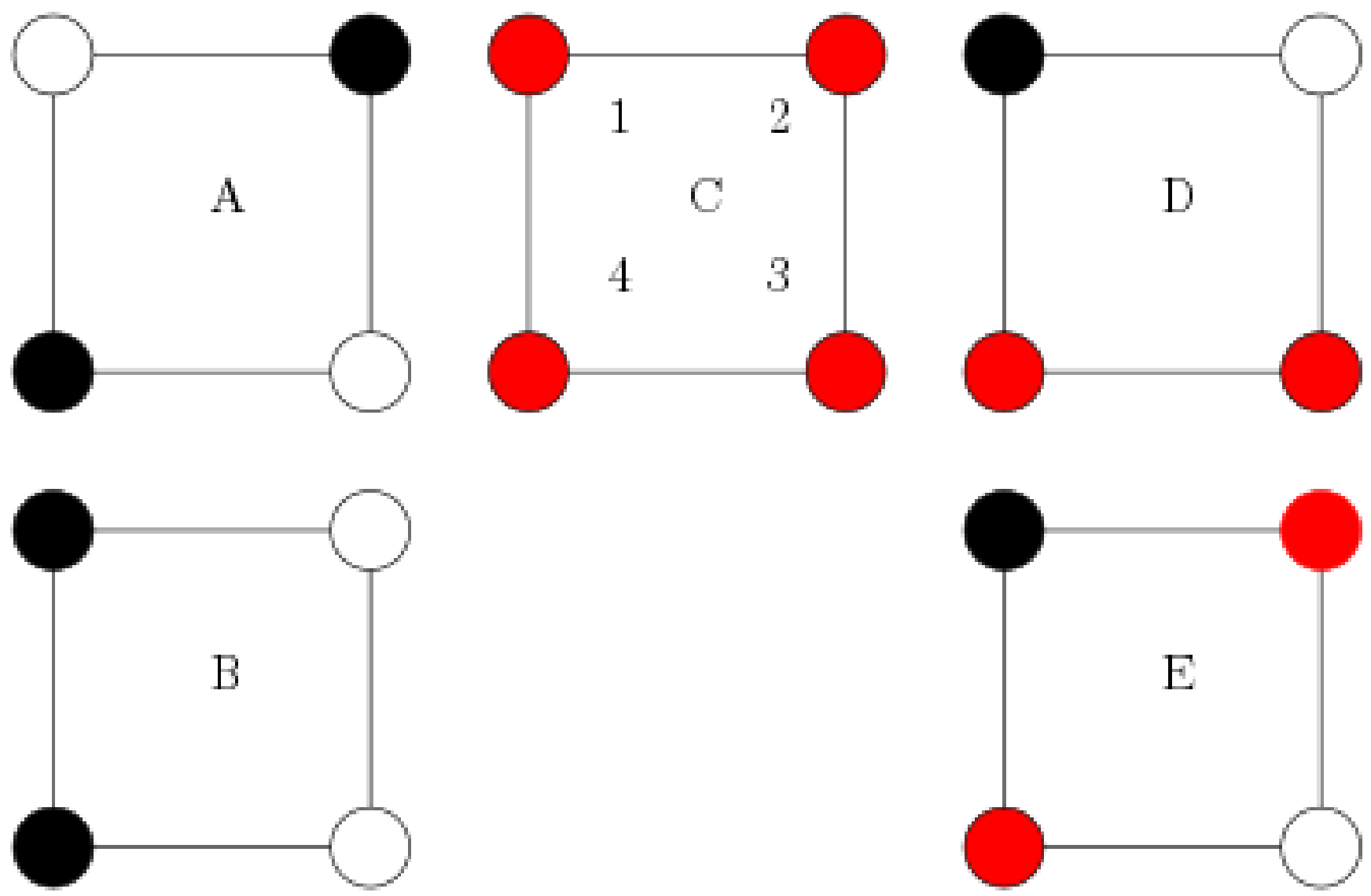

Figure 8: Atomic labelling and non-equivalent electron distributions for the four sites Hubbard model and the $\mathrm{H}_{4}$ molecule. Maximum site occupancy is restricted to two (see the text). Empty, red, and black circles indicate zero-, singly and doubly occupied sites, respectively.

There are three open-shell singlets, with symmetries $A_{1 g}, B_{1 g}$, and $B_{2 g}$, and one $A_{2 g}$ triplet state that can be formed from the $\mathrm{a}_{1 \mathrm{~g}}^{2} \mathrm{e}_{\mathrm{g}}^{2}$ ground state configuration in $\mathrm{H}_{4}$. Only the triplet can be described at the single determinant level. In the tight binding approximation, the four $\mathrm{M}_{\mathrm{S}}=0$ states can be written as linear combination of two determinants with coefficients determined by symmetry, and their EDFs and bonding indices determined algebraically. The probability distribution of the two alpha (or the equivalent two beta) electrons in these four $M_{S}=0$ states is equal: the probability that the two electrons are adjacent (B-like in Fig. 8 ) is $1 / 8$, and the probability that they occupy non- 
adjacent nodes is $1 / 4$. It is thus the different correlation pattern between the opposite spin blocks that distinguishes the four-electron EDFs in the four states. As seen in Table 1, the two-, third-, and four-center cumulants of the distribution discriminate perfectly among the states.

Table 1: two- to four-center cumulant moments of the EDF in the four $M_{S}=0$ states of the $a_{1 g}^{2} e_{g}^{2}$ configuration of $\mathrm{H}_{4}$ obtained in the tight binding approximation.

\begin{tabular}{ccccc} 
State & $\kappa_{12}=-\delta^{12} / 2$ & $\kappa_{13}$ & $\kappa_{123}$ & $\kappa_{1234}$ \\
\hline${ }^{3} \mathrm{~A}_{2 \mathrm{~g}}$ & $-1 / 8$ & $-1 / 8$ & 0 & $-3 / 64$ \\
${ }^{1} \mathrm{~B}_{2 \mathrm{~g}}$ & $-3 / 8$ & $+1 / 8$ & 0 & $-3 / 64$ \\
${ }^{1} \mathrm{~A}_{1 \mathrm{~g}}$ & $-3 / 8$ & $+1 / 8$ & 0 & $+5 / 64$ \\
${ }^{1} \mathrm{~B}_{1 \mathrm{~g}}$ & $-1 / 8$ & $-1 / 8$ & 0 & $+5 / 64$ \\
\hline
\end{tabular}

The EDFs of the Hubbard solution are not continuous at $\mathrm{U} / \mathrm{t}=0$ due to the degeneracy of the singlets. The Hubbard ground state is ${ }^{1} \mathrm{~B}_{1 \mathrm{~g}}$ at positive correlation factors, while it is the ${ }^{1} \mathrm{~A}_{1 \mathrm{~g}}$ state at negative $\mathrm{U} / \mathrm{t}$. Since these correspond to very different electron distributions, the EDF jumps at $U / t=0$. Fig. 9 shows the contribution of the $A, B, C$ and $D$ structures to the EDF in the attractive and repulsive Hubbard regimes. The $E$ structure is not populated in any of the ${ }^{1} \mathrm{~B}_{1 \mathrm{~g}},{ }^{1} \mathrm{~A}_{1 \mathrm{~g}}$ states. Fig. 10 shows the evolution of the LIs and DIs.

In the repulsive regime the $r \rightarrow \infty$ limit leads to a purely neutral $H_{4}$ structure with $p(C)=1$. As $r$ decreases to the tight binding case delocalization sets in, although in a very peculiar way: no A structures are found. In this process, the LI decreases from 1 to $5 / 8$, while the $\delta^{12}$ and $\delta^{13}$ DIs reach the same limiting value, $1 / 4$, although at different velocities. The rate of decrease of $\delta^{12}$ is clearly larger. The behavior of the ${ }^{1} \mathrm{~A}_{1 \mathrm{~g}}$ state in the attractive region is completely different. As $r \rightarrow-\infty$ only valence bond ionic structures remain, and $p(A)=1 / 3, p(B)=1 / 12$. Taking into account the two and four equivalent distributions of type $A$ and $B$, respectively, the probabilities of finding the system in any $A$ or $B$ structure tend to $2 / 3$ and $1 / 3$, respectively. These are exactly the results of an equiprobable statistical mixture of the three possible ways in which two independent $\mathrm{H}^{+} \leftrightarrow \mathrm{H}^{-}$resonances can be set up in the $\mathrm{H}_{4}$ system: $(1 \leftrightarrow 2) \wedge(3 \leftrightarrow 4),(1 \leftrightarrow 4) \wedge(2 \leftrightarrow 3)$ and $(1 \leftrightarrow 3) \wedge(2 \leftrightarrow 4)$. The $\mathrm{LI}$ of any of the $\mathrm{H}$ atoms vanishes, so we have a maximum variance situation, as in the $\mathrm{H}^{+} \leftrightarrow \mathrm{H}^{-}$limiting case of $\mathrm{H}_{2}$. It can be readily shown that $A$ and $B$ structures appear in the $(1 \leftrightarrow 2) \wedge(3 \leftrightarrow 4)$ and $(1 \leftrightarrow 4) \wedge(2 \leftrightarrow 3)$ cases, but that no B RSRSs occur in the $(1 \leftrightarrow 3) \wedge(2 \leftrightarrow 4)$ arrangement. This leads to a predominance of A resonances in the $r \rightarrow-\infty$ limit. In the two equivalent $A$ structures the electrons are found together in non-adjacent nodes, shifting 


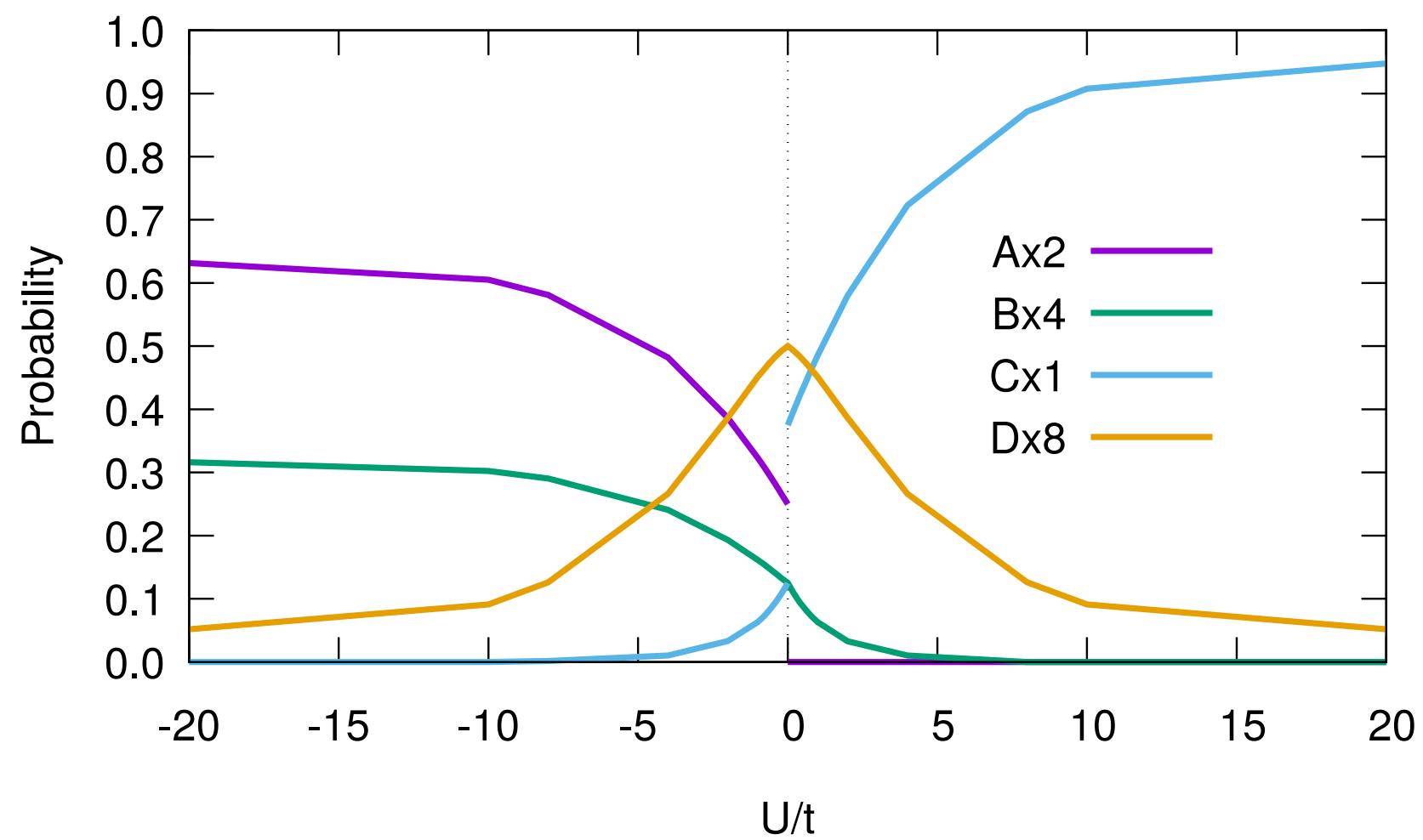

Figure 9: Probability of each RSRS for the four site Hubbard model. Notice that the multiplicities of the $A, B$, and $D$ structures are 2,4 , and 8 , respectively. The probabilities shown have been multiplied by these numbers.

simultaneously from two electrons in the 1 and 3 nodes to two electrons in 2 and 4 . This induces a positive non-adjacent covariance. If a positive fluctuation of the population is found in node 1 , a positive fluctuation also occurs in node 3 , and viceversa. The $\delta^{13}=\delta^{24}$ delocalization index has become negative. Actually, if we imagine a system in which only the A RSRSs are populated (each of the two equivalent possibilities with probabilities $1 / 2$ ), the adjacent DI would be equal to 2 and the non-adjacent $D I$ equal to -2 . This is actually the $r \rightarrow-\infty$ limit of the Hubbard ${ }^{1} B_{2 g}$ state.

These negative Dls correspond, in physical terms, to two coupled pairs of electrons that delocalize together. The concerted movement, however, may actually involve only two instead of four electrons. A model with just two electrons on nodes 1,3 with probability $1 / 2$ shifting to 2,4 with $p=1 / 2$ also displays negative non-adjacent DIs. Overall, given a single pair of electrons, the anomalous $\mathrm{DI}>1$ or $\mathrm{DI}<0$ situations involve bosonization. In the first case it is an intra-domain (intra-atomic) pair that delocalizes simultaneously to another site, while an interatomic pair of intra-atomic pairs is involved in the second case.

In short, standard weakly correlated two-center chemical bonds imply one-electron delocaliza- 


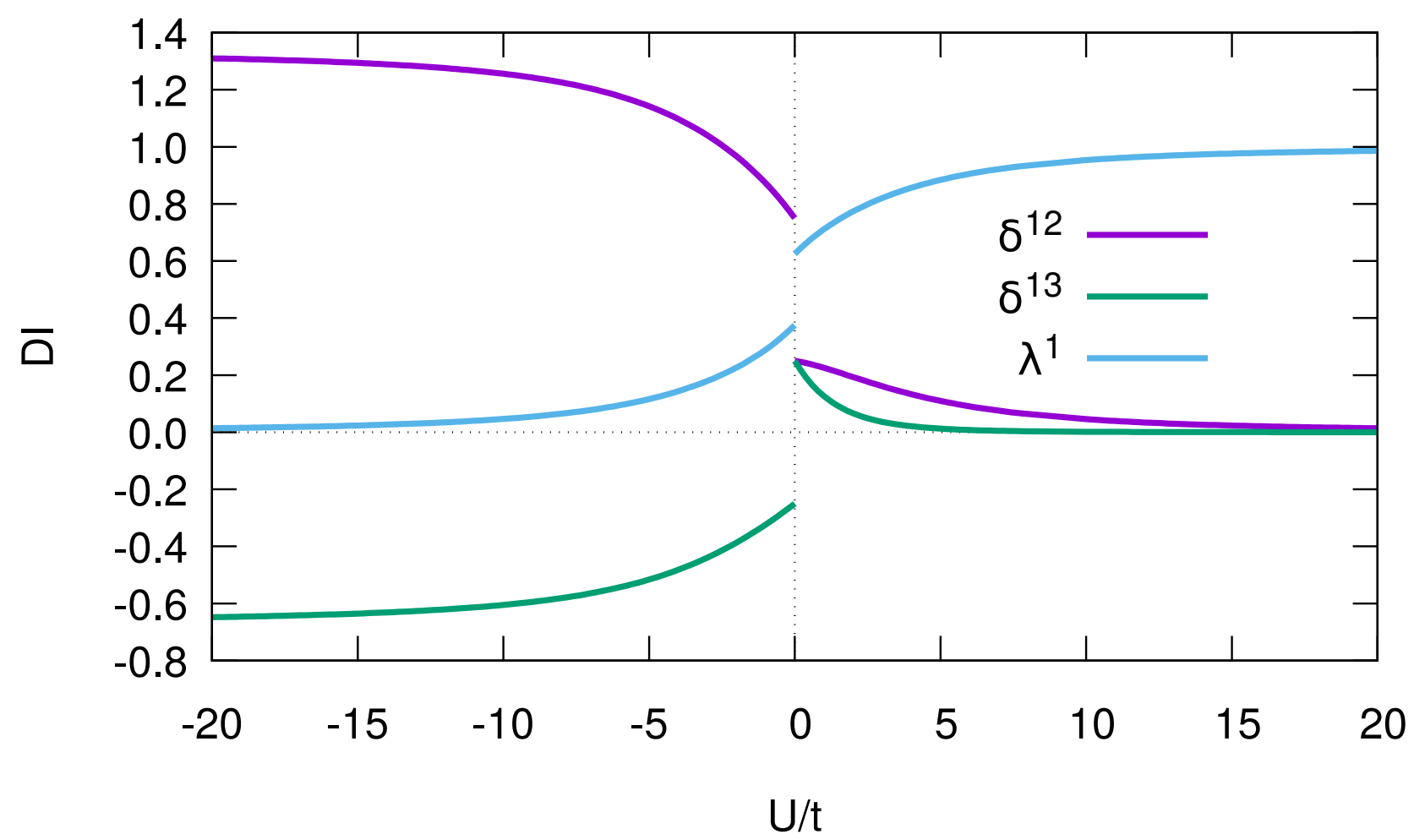

Figure 10: Localization and delocalization indices in the four sites Hubbard model. The ground state is ${ }^{1} \mathrm{~B}_{1 \mathrm{~g}} /{ }^{1} \mathrm{~A}_{1 \mathrm{~g}}$ for positive/negative $\mathrm{U} / \mathrm{t}$ values.

tion between two sites. This leads to a DI which lies between 0 and $1 / 2$. In the $f \gtrsim 0$ case the Fermi hole of this electron leaves room for an opposite spin one to occupy its same spatial region, leading to the standard Lewis pair. These two behave in an effective independent way, so the DI of the $2 c, 2 e$ link is smaller or equal to one. Contrarily, a minimum of two electrons are needed to explore new bonding landscapes. Their bosonized behavior may lead to concerted intra- or interatomic delocalization of the pair, associated to larger than one, or smaller than zero Dls, respectively.

As before, we have explored the possibility of finding these exotic behaviors in actual systems. The ground state of the $\mathrm{H}_{4}$ molecule is a $B_{1 \mathrm{~g}}$ state. [49] We have performed aug-cc-pVDZ/FCl calculations on the two first roots of each of the $B_{1 g}$ and $A_{1 g}$ symmetries. The energy profiles, with two clear conical intersections, are found in Fig. S2.

Since the attractive Hubbard $\mathrm{A}_{1 \mathrm{~g}}$ state shows negative $\delta^{13}$ values even at low $r$ values, we have examined in detail the first $A_{1 g}$ root, which displays a minimum at about $R_{e}=1.17 \AA$. As evinced by Fig. $S 2$, the barrier at $\mathrm{R} \approx 2 \AA$ is due to an avoided crossing with the third $A_{1 g}$ state. Fig. 11 shows the computed Dls and LIs. The first $\mathrm{A}_{1 \mathrm{~g}}$ state dissociates to a neutral $4 \mathrm{H}$ moiety, but the just mentioned avoided crossing makes it acquire a clear zwitterionic character at smaller distances that 
justifies the negative value of $\delta^{13}$ at $\mathrm{R}_{\mathrm{e}}$, which reaches a minimum value of -0.06 at $\mathrm{R} \approx 1.30 \AA$. The neutral character is recovered at even smaller distances after the conical intersection with the second state. This interesting behavior is even more clear after examining the EDF.

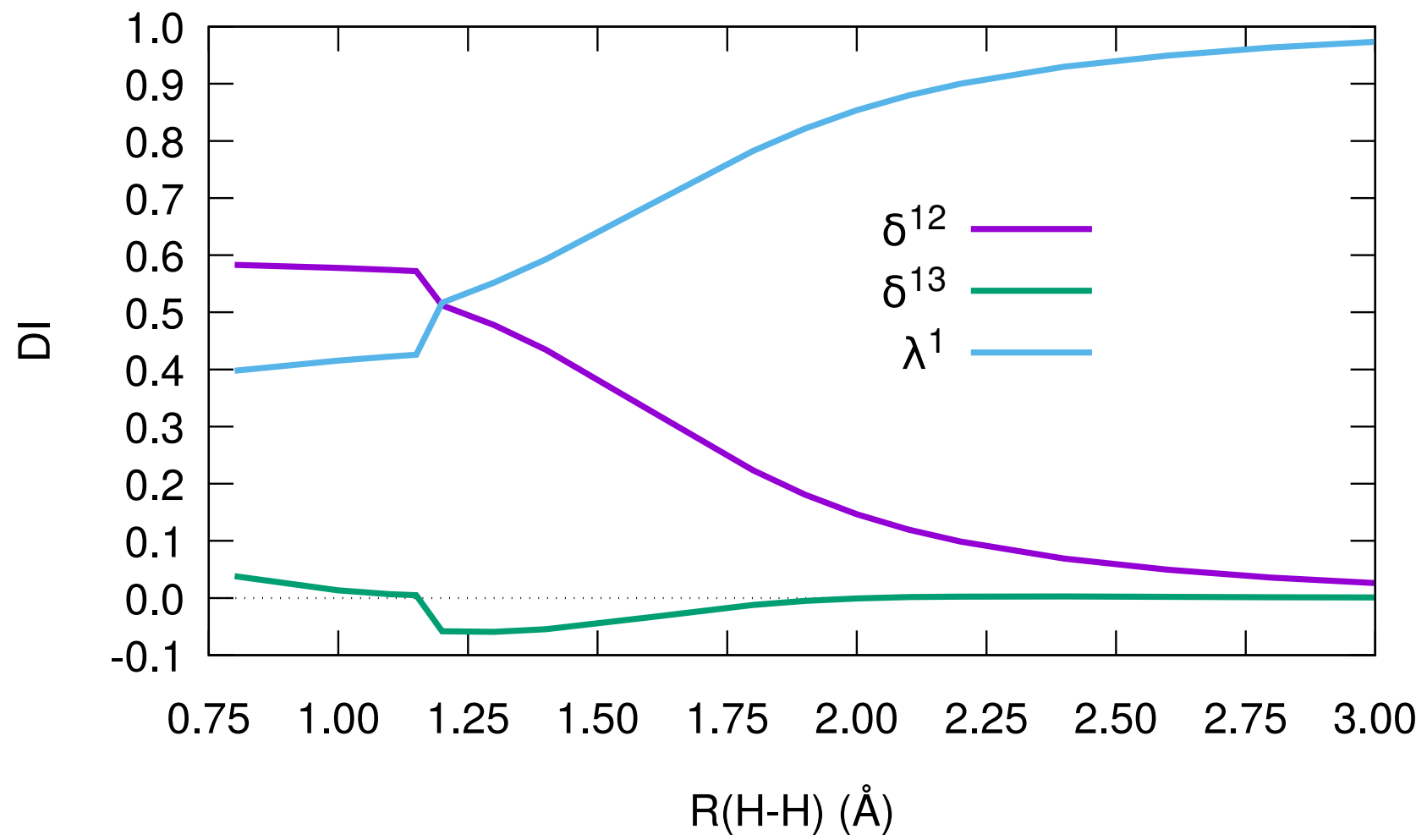

Figure 11: Localization and delocalization indices in the $\mathrm{FCl}$ calculation of the lowest ${ }^{1} \mathrm{~A}_{1 \mathrm{~g}}$ state of $\mathrm{H}_{4}$.

Fig 12 shows the evolution of the probability of the six main RSRSs of the lowest ${ }^{1} \mathrm{~A}_{1 \mathrm{~g}}$ state. Notice how the onset of delocalization as $\mathrm{R}$ is shortened couples the decrease of the weight of the neutral $C$ structure with the increase of that of $D$, much as in the repulsive Hubbard model, and how at $\mathrm{R} \approx 2.0 \AA$ after the avoided crossing, the A structure appears, being third in rank as in the attractive Hubbard results shown before. The intersection with the second $A_{1 g}$ root at a distance close to the $\mathrm{R}_{\mathrm{e}}$ has a big impact on the probability distribution. $\delta^{13}$ becomes positive again through the exchange. It is thus the crossing with states that dissociate into ionic resonances that forces the appearance of negative Dls in neutrally dissociating states. 


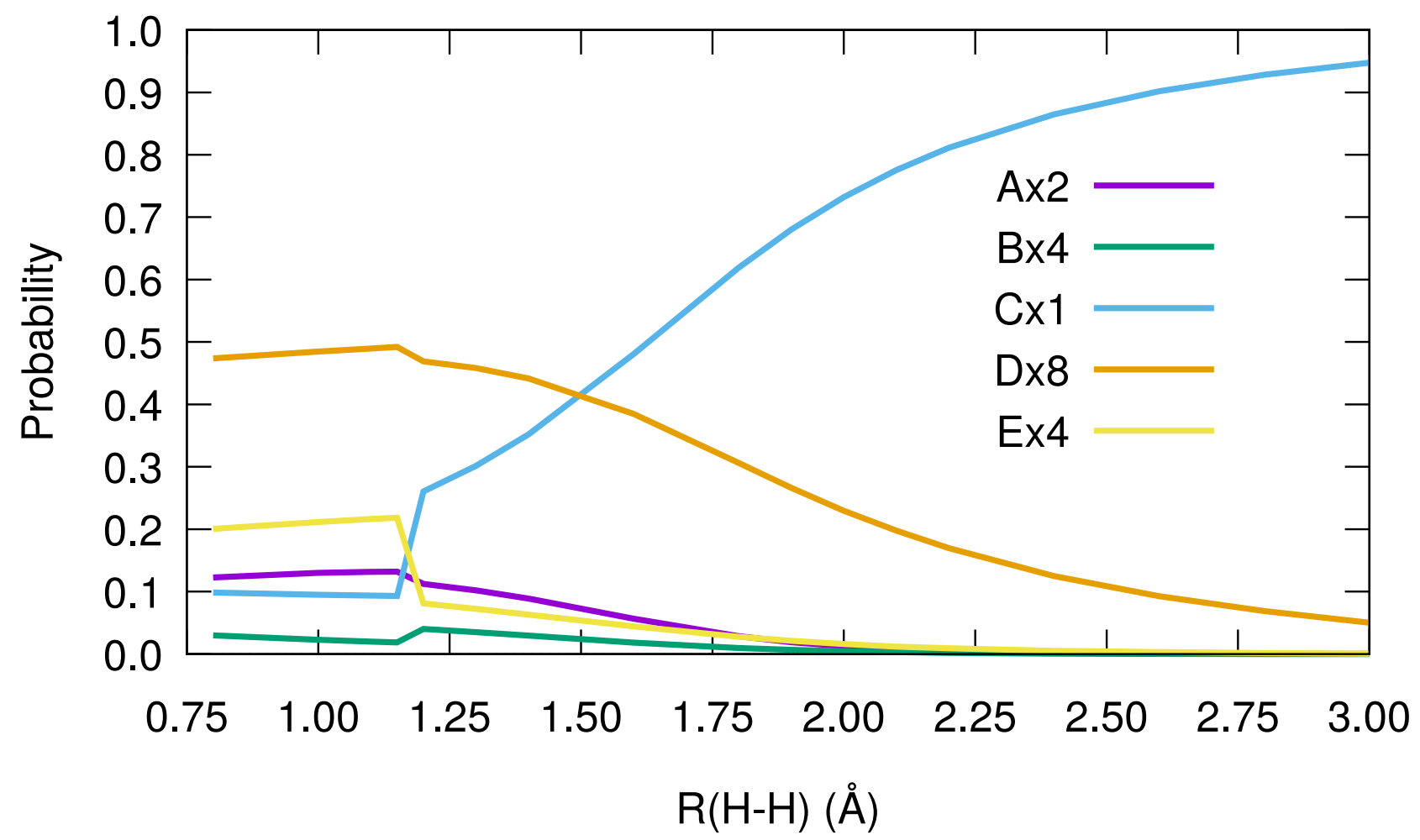

Figure 12: Evolution of the EDF in the $\mathrm{FCl}$ calculation of the lowest ${ }^{1} \mathrm{~A}_{1 \mathrm{~g}}$ state of $\mathrm{H}_{4}$ with the shortest internuclear distance $\mathrm{R}$.

\section{The conical intersection in $\mathrm{C}_{2} \mathrm{H}_{4}$}

In our previous work, [11] we have examined the energy redistribution at the minimum energy conical intersection associated with the twisted-pyramidalized geometry of ethylene. We now analyze the EDFs along the minimum energy path (MEP) of the first valence excited state (V). The main geometrical changes are the torsion and pyramidalization of the methylene fragments, and to a lesser extent the $\mathrm{H}, \mathrm{H}$ scissoring and the $\mathrm{C}-\mathrm{C}$ streching/compression, in agreement with previous reports. [50] The barrierless MEP (Fig. S1) connects the Franck-Condon region with the conical intersection seam which involves the ground state $(\mathrm{N})$. Although several Rydberg states are superimposed on the initial portion of the MEP, they were omitted for simplicity from the present discussion.

The analysis of the EDFs along the MEP provides detailed information about the main changes in electronic structure from the excitation region to the point where the crossing between the $\mathrm{V}$ and $\mathrm{N}$ surfaces takes place. Figs. 13 and 14 show the evolution of the $\mathrm{CH}_{2}-\mathrm{CH}_{2}$ DI and EDF contributions. It stands out clear that all relevant electronic redistribution between the two groups occur during the twisting phase up to the conical intersection at MEP $\approx 4.21$ au. The correlated DI of the ground 
state is 1.63 , a typical value for a double bond. Interestingly, the DIs of both the $\mathrm{V}$ and $\mathrm{Z}$ states are considerably greater than that of S0, pointing to a clearly zwitterionic component. The EDFs evince that the probability of the $(8,8)$ RSRS of the $V$ and $Z$ states are significantly smaller than that of the ground state, and that the contrary is true for the $(6,10)$ and $(10,6)$ RSRSs. Interestingly, the singleelectron exchange $(7,9)$ and $(9,7)$ RSRSs are almost unaltered during the twisting phase. This implies that one out of the two links has remained basically unaltered, while the other has shifted to the $f<0$ regime as shown in the following. We have approximately decomposed the EDF of the $V$ state at the $\mathrm{N}$ equilibrium geometry, with $\delta=2.31$, into two independent links. [21] This leads to two symmetrical two-center two-electron links. The first one with $f=0.06$ is a normal bond, while the second, with $\mathrm{f}=-0.29$ and $\delta=1.29$ is clearly bosonized.

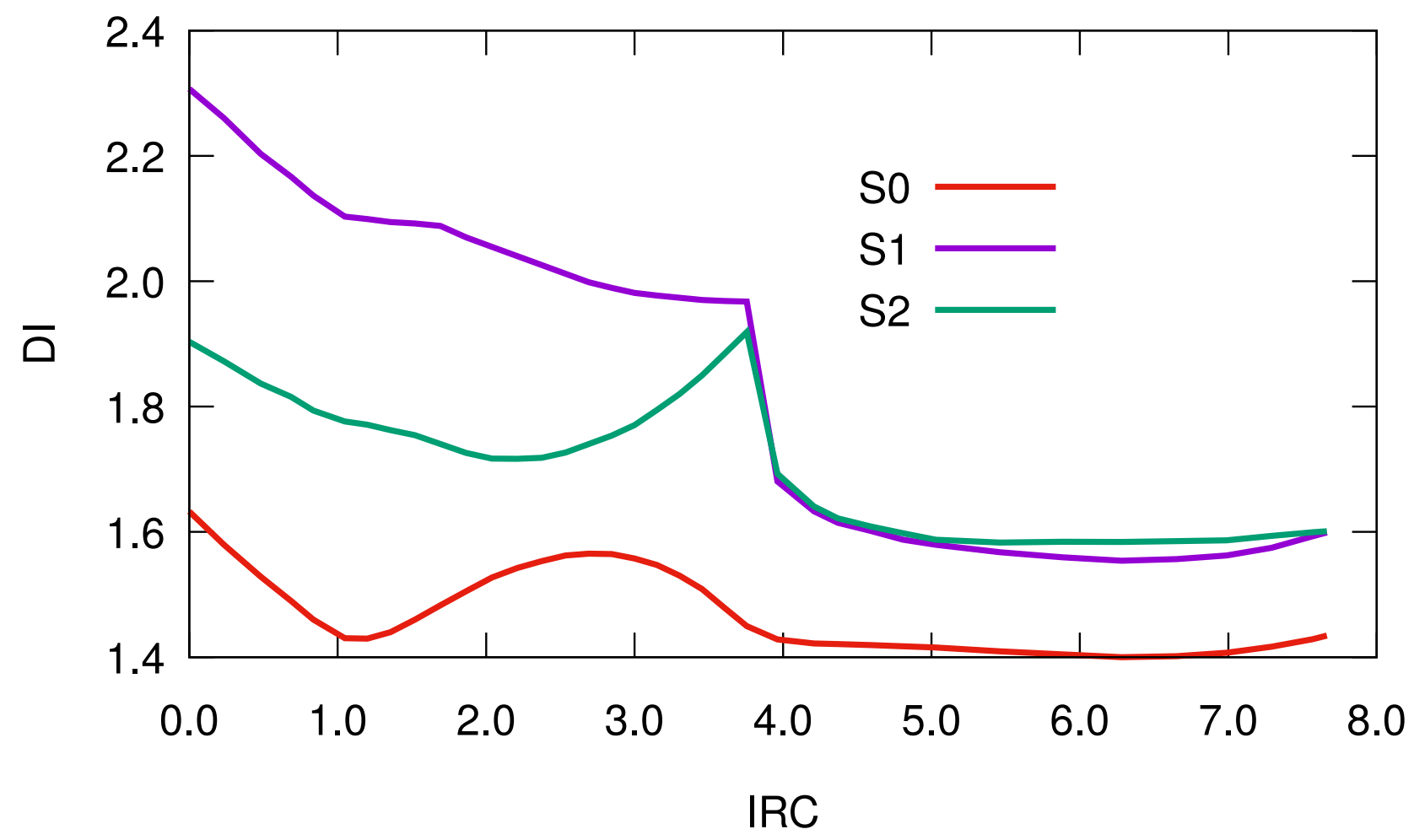

Figure 13: Evolution of the Dls between the methylene groups of the $\mathrm{N}, \mathrm{V}$, and $\mathrm{Z}$ states in ethylene along the IRC described in the text. All data in au.

It is also interesting to remark, although we will not examine it in more detail, that after the Z/V intersection, during the pyramidalization step, the two methylene fragments cease to be equivalent, and that a rather clear charge separation from one to the other is observed, particularly in the $Z$ state, a behavior that has been referred to as sudden polarization. [51] 


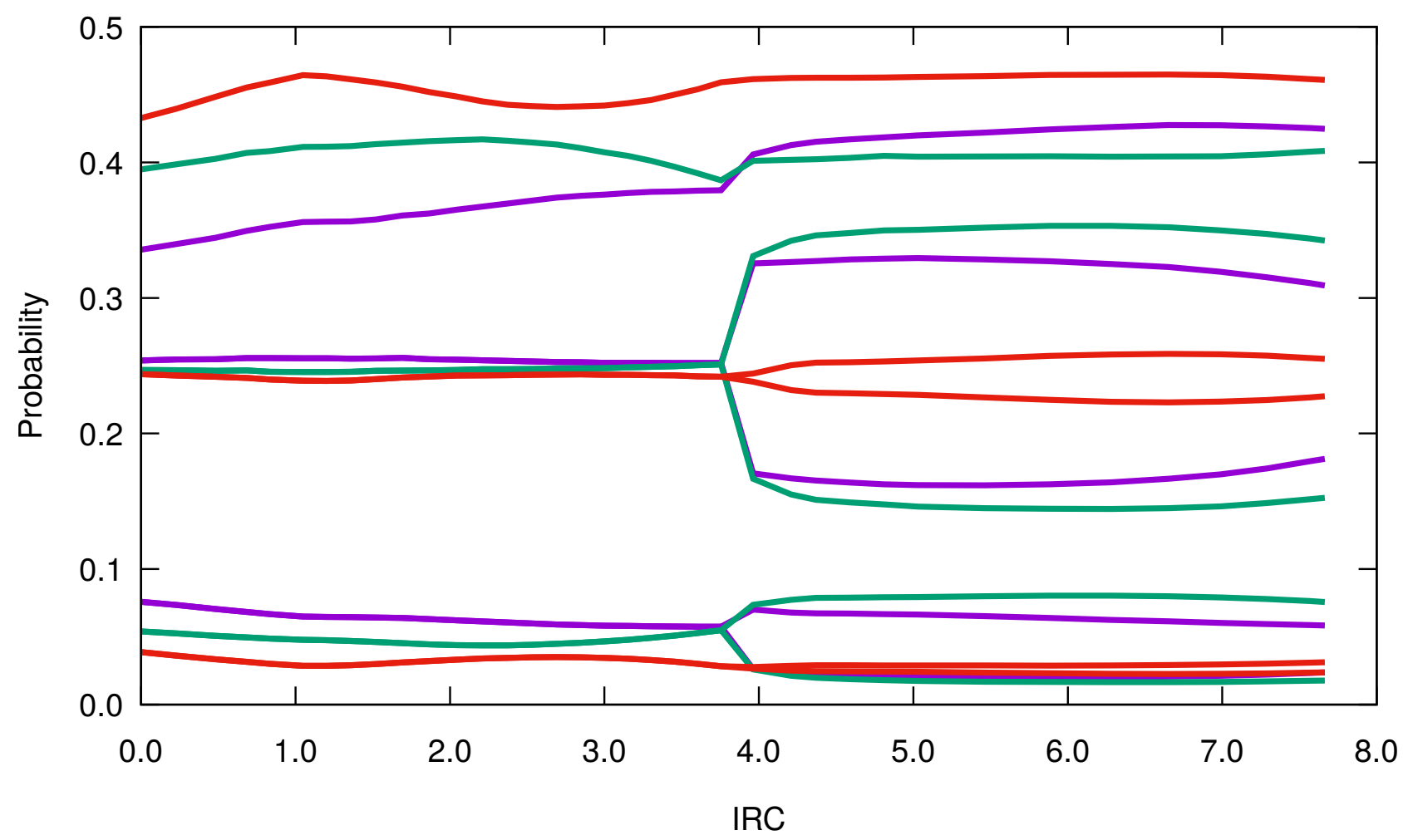

Figure 14: EDF of the $\mathrm{CH}_{2}-\mathrm{CH}_{2}$ groups in the $\mathrm{N}$ (red), $\mathrm{V}$ (blue), and $\mathrm{Z}$ (green) states of ethylene along the IRC described in the text. All data in au. The lower bunch describes the $(6,10)$ and $(10,6)$ pairs of RSRS, the middle bunch the $(7,9)$ and $(9,7)$ pairs, and the upper one the neutral $(8,8)$ structure.

\section{Conclusions}

We have shown in this work how the investigation of real space descriptors and electron counting techniques may be used to enlarge our knowledge about the types and nature of the chemical bonds found in excited states.

Electron distribution functions provide the full statistical distribution of the electron populations in real space regions. When an atomic partition like that provided by the QTAIM is chosen, as we have done here, it has been shown before that basically all modern chemical bonding descriptors can be interpreted in terms of the statistical fluctuation of the electron populations. We have focused on one and two-center bond indices, like the localization (LI) and delocalization (DI) indices. By using physical models it is shown that the full landscape of bond orders, for instance, is considerably larger than imagined, and that even for two electron systems, positive but greater than one, or even negative DIs, are in principle possible. Both cases are related to a strong coupling of pairs of electrons, which behave and delocalize like a single pair. This implies a kind of gregarious, i.e. 
bosonic, behavior of the electronic system.

The DI greater than one case has been related to valence bond ionic resonances, or zwitterionic states, which abound in excited states in which two intra-atomic strongly coupled electrons delocalize simultaneously. We have demonstrated the existence of $\delta>1$ situations for two-electron systems in several excited states of the hydrogen molecule which are modeled within an attractive two-sites Hubbard model. This indicates that the bosonization of an electron pair is a process that can be readily observed in simple chemical systems. A more interesting case of this kind of bosonization, the excited states of ethylene, shows that this finding is not only of academic interest.

We have also shown that strong standard bonds, like those in excimers (some excimer states of $\mathrm{He}_{2}$ have been used as examples) may hide two different 2c,1e delocalization channels. This is easily uncovered through the use of natural adaptive orbitals. Similarly, avoided crossings between neutral (covalent) and charge transfer (ionic) states in highly ionic molecules like LiF or LiH have also been examined, uncovering how in extreme cases they correspond to mirror states with respect to electron jump that exhibit the same bond orders.

Finally, negative DI bonding patterns demand the existence of a third center or site. We show that a cyclic four sites repulsive/attractive Hubbard model gives rise easily to negative Dls between non-adjacent, i.e. diagonal, nodes. It corresponds, physically, to a pair of strongly coupled electrons lying on two different sites that delocalize together (to the other diagonal in the four sites square). $\mathrm{FCl}$ calculations in one excited state of the $\mathrm{H}_{4}$ molecule corroborate the existence of these exotic situations.

This closes the loop with two electrons. We have the following simplified scenario. In the weak correlation regime, an electron may delocalize to an available site (domain) leading to a 2c,1e link with positive DI, strictly smaller than $1 / 2$. Another opposite spin electron may occupy its Fermi hole to form a standard Lewis pair, which should be understood as two independent 2c,1e links leading the $2 \mathrm{c}, 2 \mathrm{e}$ paradigm with a DI smaller than one. If two electrons are strongly coupled with positive correlation this leads to localization and bonding inhibition (associated to a Mott transition in the thermodynamic limit). If the pair is coupled through negative correlation, it may behave as a unit lying on one site that delocalizes to an available domain (a zwitterionic pattern) giving rise to a DI greater than one, or the pair may reside in two different sites, delocalizing together to available regions. In this case we find negative Dls. 
Further analyses as the number of centers and/or coupled electrons increases are obviously possible. We think they should clearly be investigated.

\section{Acknowledgements}

We thank the spanish MINECO, grant PGC2018-095953-B-I00, the FICyt, grant IDI-2018-000177 and the European Union FEDER funds for financial support. J. H.-T. also thanks supercomputer resources (project LANCAD-UNAM-DGTIC-103) and PAIP Facultad de Química UNAM (grant 50009004).

\section{References}

[1] J. J. W. McDouall, Computational Quantum Chemistry: Molecular Structure and Properties in Silico, of RSC theoretical and computational chemistry series, Royal Society of Chemistry, 2013.

[2] A. Stoneham, Physica B: Condensed Matter 2003, 340-342, 48 - 57, Proceedings of the 22nd International Conference on Defects in Semiconductors.

[3] M. B. Smith, J. Michl, Chem. Rev. 2010, 110, 6891-6936.

[4] E. Sjulstok, J. M. Olsen, I. A. Solov'yov, Sci. Rep. 2015, 5, 18446.

[5] C. A. Brautigam, B. S. Smith, Z. Ma, M. Palnitkar, D. R. Tomchick, M. Machius, J. Deisenhofer, Proc. Natl. Acad. Sci. 2004, 101, 12142-12147.

[6] M. Staniforth, V. G. Stavros, Proc. R. Soc. London A 2013, 469, 20130458.

[7] A. K. Dutta, N. Vaval, S. Pal, Int. J. Quantum Chem 2018, 118, 25594-25606.

[8] E. Runge, E. K. U. Gross, Phys. Rev. Lett. 1984, 52, 997-1000.

[9] G. M. Barca, A. T. Gilbert, P. M. Gill, J. Chem. Theory Comput. 2018, 14, 1501-1509.

[10] R. F. W. Bader, Atoms in Molecules, Oxford University Press, Oxford, 1990. 
[11] J. Jara-Cortés, J. M. Guevara-Vela, Á. Martín Pendás, J. Hernández-Trujillo, J. Comput. Chem. 2017, 38, 957-970.

[12] M. A. Blanco, A. Martín Pendás, E. Francisco, J. Chem. Theory Comput. 2005, 1, 1096-1109.

[13] E. Francisco, A. Martín Pendás, M. A. Blanco, J. Chem. Theory Comput. 2006, 2, 90-102.

[14] C. Outeiral, M. A. Vincent, Á. M. Pendás, P. L. A. Popelier, Chem. Sci. 2018, 9, 5517-5529.

[15] A. M. Pendás, E. Francisco, Phys. Chem. Chem. Phys. 2018, 20, 16231-16237.

[16] M. Giambiagi, M. S. de Giambiagi, K. C. Mundim, Struct. Chem. 1990, 1, 423-427.

[17] K. C. Mundim, M. Giambiagi, M. S. de Giambiagi, J. Phys. Chem. 1994, 98, 6118-6119.

[18] R. Ponec, P. Bultinck, A. Gallegos-Saliner, J. Phys. Chem. A 2005, 109, 6606-6609.

[19] M. Mandado, M. J. González-Moa, R. A. Mosquera, J. Comput. Chem. 2007, 28, 127-136.

[20] E. Matito, M. Solà, P. Salvador, M. Duran, Faraday Discuss. 2007, 135, 325-345.

[21] A. Martín Pendás, E. Francisco, M. A. Blanco, Phys. Chem. Chem. Phys. 2007, 9, 1087-1092.

[22] F. H. L. Essler, H. Frahm, F. Göhmann, A. Klümper, V. Korepin, The One-Dimensional Hubbard Model, Cambridge, Cambridge., 2005.

[23] E. Francisco, A. M. Pendás, M. Blanco, Comput. Phys. Commun. 2008, 178, 621 - 634.

[24] E. Francisco, A. Martín Pendás, M. A. Blanco, J. Chem. Phys. 2007, 126, 094102.

[25] E. Francisco, A. Martín Pendás, M. García-Revilla, R. Álvarez Boto, Comput. Theor. Chem. 2013, 1003, 71-78.

[26] K. B. Wiberg, Tetrahedron 1968, 24, 1083-1096.

[27] I. Mayer, Chem. Phys. Lett. 1983, 97, 270-274.

[28] M. Menéndez, R. Álvarez Boto, E. Francisco, Á. Martín Pendás, J. Comput. Chem. 2015, 36, 833-843. 
[29] M. Rafat, P. L. A. Popelier in The Quantum Theory of Atoms in Molecules, Wiley-VCH Verlag GmbH \& Co. KGaA, Weinheim, Germany, 2007, pp. 121-140.

[30] E. Francisco, D. Menéndez-Crespo, A. Costales, A. Martín Pendás, J. Comput. Chem. 2017, 38, 816-829.

[31] A. Martín Pendás, E. Francisco, Phys. Chem. Chem. Phys. 2018, 20, 16231-16237.

[32] L. Gagliardi, P. Pyykkö, B. Roos, Phys. Chem. Chem. Phys. 2005, 7, 2415-2417.

[33] E. H. Lieb, F. Y. Wu, Phys. Rev. Lett. 1968, 20, 1445-1448.

[34] A. Gallo-Bueno, M. Kohout, A. Martín Pendás, J. Chem. Theory Comput. 2016, 12, 3053-3062.

[35] J. P. Carbotte, Rev. Mod. Phys. 1990, 62, 1027-1157.

[36] Q. Sun, T. C. Berkelbach, N. S. Blunt, G. H. Booth, S. Guo, Z. Li, J. Liu, J. D. McClain, E. R. Sayfutyarova, S. Sharma, S. Wouters, G. K.-L. Chan, Wiley Interdiscip. Rev. Comput. Mol. Sci. 2018, 8, 1340.

[37] T. H. Dunning, J. Chem. Phys. 1989, 90, 1007-1023.

[38] H.-J. Werner, P. J. Knowles, G. Knizia, F. R. Manby, M. Schütz, Wiley Interdiscip. Rev. Comput. Mol. Sci. 2012, 2, 242-253.

[39] T. Shiozaki, Wiley Interdiscip. Rev. Comput. Mol. Sci. 2018, 8, e1331.

[40] A. Martín Pendás, E. Francisco, PROMOLDEN. A QTAIM/IQA code (available from the authors upon request at ampendas@uniovi.es).

[41] E. Francisco, Denmat. A cumulant analysis code (available from the author upon request at evelio@uniovi.es).

[42] T. Sharp, At. Data Nucl. Data Tables 1970, 2, 119 - 169.

[43] L. W. Chun, Y. Gim, T. H. Choi, Bull. Korean Chem. Soc. 2013, 34, 1771-1779.

[44] M. García-Revilla, P. L. A. Popelier, E. Francisco, A. Martín Pendás, J. Chem. Theory Comput. 2011, 7, 1704-1711. 
[45] S. Shaik, D. Danovich, W. Wu, P. C. Hiberty, Nat. Chem. 2009, 1, 443-449.

[46] W. Wu, J. Gu, J. Song, S. Shaik, P. C. Hiberty, Angew. Chem. Int. Ed. 2009, 48, 1407-1410.

[47] S. Yang, A. M. Ellis, Chem. Soc. Rev. 2013, 42, 472-484.

[48] S. E. Huber, A. Mauracher, J. Phys. Chem. A 2014, 118, 6642-6647.

[49] J. K. Ellis, R. L. Martin, G. E. Scuseria, J. Chem. Theory Comput. 2013, 9, 2857-2869.

[50] M. Barbatti, J. Paier, H. Lischka, J. Chem. Phys. 2004, 121, 11614-11624.

[51] A. Viel, R. P. Krawczyk, U. Manthe, W. Domcke, Angew. Chem. Int. Ed. 2003, 42, 3434-3436. 